\title{
Deconstructing the Polymerase Chain Reaction II: An improved workflow and effects on artifact formation and primer degeneracy
}

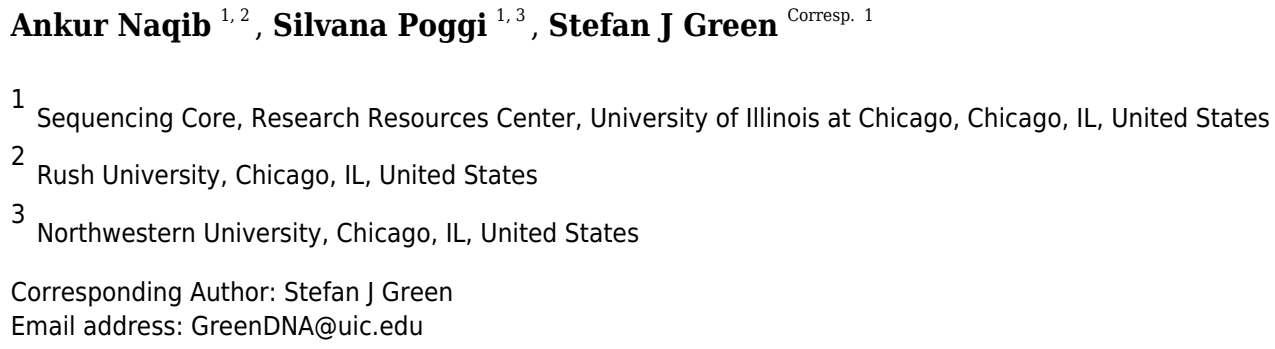

Polymerase chain reaction (PCR) amplification of complex microbial genomic DNA templates with degenerate primers can lead to distortion of the underlying community structure due to inefficient primer-template interactions leading to bias. We previously described a method of deconstructed PCR ("PEX PCR") to separate linear copying and exponential amplification stages of PCR to reduce PCR bias. In this manuscript, we describe an improved deconstructed PCR ("DePCR") protocol separating linear and exponential stages of PCR and allowing higher throughput of sample processing. We demonstrate that the new protocol shares the same benefits of the original and show that the protocol dramatically and significantly decreases the formation of chimeric sequences during PCR. By employing PCR with annealing temperature gradients, we further show that there is a strong negative correlation between annealing temperature and the evenness of primer utilization in a complex pool of degenerate primers. Shifting primer utilization patterns mirrored shifts in observed microbial community structure in a complex microbial DNA template. We further employed the DePCR method to amplify the same microbial DNA template independently with each primer variant from a degenerate primer pool. The non-degenerate primers generated a broad range of observed microbial communities, but some were highly similar to communities observed with degenerate primer pools. The same experiment conducted with standard PCR led to consistently divergent observed microbial community structure. The DePCR method is simple to perform, is limited to PCR mixes and cleanup steps, and is recommended for reactions in which degenerate primer pools are used or when mismatches between primers and template are possible. 
1 Deconstructing the Polymerase Chain Reaction II: An improved workflow and effects on

2 artifact formation and primer degeneracy

3

4 Running title: Deconstructed PCR

5

6 Ankur Naqib ${ }^{\mathbf{1}}{ }^{2} \%$, Silvana Poggi ${ }^{1 \wedge}$, Stefan J. Green*1

7 Author affiliations: ${ }^{1}$ Sequencing Core, Research Resources Center, University of Illinois at

8 Chicago, Chicago, IL; ${ }^{2}$ Department of Bioengineering, University of Illinois at Chicago,

9 Chicago, Illinois, USA.

$10 \%$ Current address: Rush University, Chicago, IL

$11 \wedge$ Current address: Northwestern University, Chicago, IL

13 Corresponding author: Stefan J. Green (GreenDNA@uic.edu); 835 S. Wolcott, A-310, 14 Chicago, Illinois, 60612 
Abstract

Polymerase chain reaction (PCR) amplification of complex microbial genomic DNA templates with degenerate primers can lead to distortion of the underlying community structure due to inefficient primer-template interactions leading to bias. We previously described a method of deconstructed PCR ("PEX PCR") to separate linear copying and exponential amplification stages of PCR to reduce PCR bias. In this manuscript, we describe an improved deconstructed PCR ("DePCR") protocol separating linear and exponential stages of PCR and allowing higher throughput of sample processing. We demonstrate that the new protocol shares the same benefits of the original and show that the protocol dramatically and significantly decreases the formation of chimeric sequences during PCR. By employing PCR with annealing temperature gradients, we further show that there is a strong negative correlation between annealing temperature and the evenness of primer utilization in a complex pool of degenerate primers. Shifting primer utilization patterns mirrored shifts in observed microbial community structure in a complex microbial DNA template. We further employed the DePCR method to amplify the same microbial DNA template independently with each primer variant from a degenerate primer pool. The non-degenerate primers generated a broad range of observed microbial communities, but some were highly similar to communities observed with degenerate primer pools. The same experiment conducted with standard PCR led to consistently divergent observed microbial community structure. The DePCR method is simple to perform, is limited to PCR mixes and cleanup steps, and is recommended for reactions in which degenerate primer pools are used or when mismatches between primers and template are possible. 


\section{Introduction}

The small subunit (SSU) ribosomal RNA (rRNA) gene is the most frequently targeted gene in studies of complex microbial systems. A common approach for microbial community studies is to extract genomic DNA (gDNA) from multiple samples, amplify gDNA by PCR using locus-specific SSU rRNA gene primers containing sequencing adapters and a sample-specific barcode, and equimolar pooling and sequencing [1]. A number of major caveats are associated with such an approach: (i) Microorganisms contain a variable number of rRNA operons [2, 3] and analyses of rRNA genes present a distorted representation of relative cellular abundance; (ii) PCR primer pools are often degenerate or the primers are anticipated to anneal to template sequences containing mismatches with the primers, thereby producing bias in amplification efficiency among different templates; and (iii) samples are generally heavily amplified (30 cycles or more) leading to a distortion of the template proportions in the original mixtures and to the possibility of extensive chimera formation.

Recently, we identified a novel source of PCR bias - namely, the simultaneous operation of linear copying and exponential amplification during the early cycles of PCR with degenerate primers [4]. We hypothesized that primer-genomic DNA template annealing operates at a different, and likely lower, efficiency compared to primer-amplicon annealing. These primertemplate interactions, operating at different efficiencies, both contribute to distortion of the underlying template community, particularly in the early cycles of PCR. To address this source of bias, we developed the polymerase-exonuclease (PEX) PCR method to separate PCR into two distinct stages of linear copying and exponential amplification. Furthermore, the PEX PCR method prevents the locus-specific primers from interacting with gDNA template after the first two cycles of linear copying. Although effective, the PEX PCR method requires an enzymatic 
59 step (exonuclease), which lengthens the workflow. We sought to improve upon the prior protocol

60 and remove the effort associated with exonuclease treatment. Nonetheless, the PEX PCR method

61 - and the separation of linear copying and exponential amplification - serves as the conceptual

62 foundation for the new method. In PEX PCR, after two cycles of linear amplification with locus-

63 specific primers containing 5' non-degenerate linker sequences, the initial stage of the reaction is

64 terminated, primers are removed with exonuclease I treatment, and the linear copies

65 subsequently amplified using non-degenerate primers targeting the 5 ' linker sequences (Figure

1). Here, we present a method that replaces exonuclease treatment with size-selective bead-based

67 purification (e.g. AMPure XP beads) but achieves substantial savings in overall labor and sample manipulation by a pooling of all samples prior to purification. We note that the strategy of linear copying followed by exponential amplification using primers targeting linker sequences has been employed previously for sequence-independent amplification of whole genome or metagenome DNA using random primers in place of locus-specific primers [5]. PEX PCR concept, while retaining the ability to reduce PCR bias. To demonstrate the effectiveness of the updated workflow, we replicated a temperature-gradient analysis of a single complex environmental genomic DNA sample using both standard PCR and DePCR workflows. Data were interrogated to examine the observed microbial community structure by method and reaction annealing temperature. In addition, primer utilization profiles (PUPs) were analyzed to assess the effects of annealing temperature on the relative utilization of each primer within a with varying input gDNA. A final experiment examined the ability of each unique primer within 
81 a degenerate primer pool to amplify a complex environmental sample using both the standard

82 PCR and DePCR methodologies.

83

84

85

86

87

88

89

90

91

92

93

94

95

96

97

98

99

100

101

\section{Materials and Methods}

DNA Templates

6

A single microbial genomic DNA (gDNA) sample obtained from chinchilla feces was used for this study. Chinchilla feces were acquired from the cage of pet animals and the sample serves as a representative complex microbial community. No specific permissions were required for collection of the chinchilla feces. The fecal sample was extracted using the PowerSoil DNA extraction kit (Mo Bio Laboratories, Carlsbad, CA).

\section{Primer Synthesis}

The primers used for this study are 341F (CCTACGGGAGGCAGCAG) [6, 7] and 806R

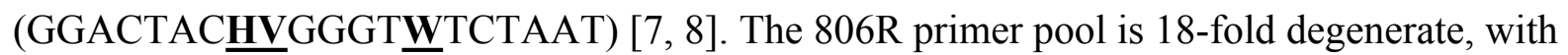
theoretical melting temperatures ranging from $54.7^{\circ} \mathrm{C}$ to $61^{\circ} \mathrm{C}$. Melting temperatures of the primers were calculated using the OligoAnalyzer3.1 tool [9], assuming $250 \mathrm{nM}$ primer concentration, $2 \mathrm{mM} \mathrm{Mg}^{2+}$, and $0.2 \mathrm{mM}$ dNTPs. Synthesis of the primers was performed either as single degenerate primer pools (standard approach), or as individual primers without degeneracies by Integrated DNA Technologies (IDT; Coralville, IA). Primers were synthesized as LabReady and ordered at a fixed concentration of 100 micromolar. Primers contained common sequence linkers (CS1 and CS2) at the 5' ends, as shown in Table 1. Linker sequences 
102 are required for the later incorporation of Illumina sequencing adapters and sample-specific 103 barcodes.

The standard PCR protocol or targeted amplicon sequencing (TAS) protocol is a twostage NGS library preparation protocol for generating barcoded amplicons ready for Illumina sequencing, and was performed as described previously [10] (Figure 1A). Briefly, gDNA was amplified by PCR with primers CS1_341F and CS2_806R. The first stage PCR reaction was conducted in a total reaction volume of $10 \mu \mathrm{l}$. Each reaction contained $5 \mu \mathrm{l}$ of MyTaq HS master mix (Bioline, Taunton, MA), $0.5 \mu 1$ of each primer or degenerate primer at a concentration of 5 $\mu \mathrm{M}$ (e.g., CS1_341F and CS2_806R; leading to a $250 \mathrm{nM}$ working concentration), $10 \mathrm{ng}$ of gDNA template, and water up to $10 \mu 1$ total volume. The first stage of the PCR was conducted using the following thermocycling conditions: $95^{\circ} \mathrm{C}$ for 5 minutes, followed by 28 cycles of $95^{\circ} \mathrm{C}$ for 30 seconds, annealing temperature (from $40^{\circ} \mathrm{C}$ to $60^{\circ} \mathrm{C}$ ) for 30 seconds, $72^{\circ} \mathrm{C}$ for 30 seconds; and a final elongation step at $72^{\circ} \mathrm{C}$ for 7 minutes. Subsequently, a second PCR amplification was performed in $10 \mu 1$ reactions in 96-well plates to incorporate Illumina sequencing adapters and a sample-specific barcode. A mastermix for the entire plate was made using the MyTaq HS 2X mastermix. Each well received a separate primer pair with a unique 10base barcode, obtained from the Access Array Barcode Library for Illumina (Fluidigm, South

121 San Francisco, CA; Item\# 100-4876). These Access Array primers contained the CS1 and CS2

122 linkers at the $3^{\prime}$ ends of the oligonucleotides. One $\mu l$ of reaction mixture from the first stage amplification was used as input template for the second stage reaction, without cleanup. Cycling conditions were as follows: $95^{\circ} \mathrm{C}$ for 5 minutes, followed by 8 cycles of $95^{\circ} \mathrm{C}$ for $30^{\prime}, 60^{\circ} \mathrm{C}$ for 
12530 " and $72{ }^{\circ} \mathrm{C}$ for 30 ”. A final, 7-minute elongation step was performed at $72{ }^{\circ} \mathrm{C}$. Samples were 126 pooled and sequenced on an Illumina MiSeq employing V2 chemistry and 2x250 base reads.

\section{Deconstructed PCR (DePCR) Protocol}

As with the TAS method, the DePCR method is also a two-stage PCR process (Figure

1C) and is a modification of the previously described PEX PCR method (Figure 1B). For each

sample, the first stage reaction of DePCR (four total cycles) was conducted in a 96-well plate

with each well containing $5 \mu \mathrm{l}$ of MyTaq master mix, $0.5 \mu 1$ of each primer or degenerate primer

at a concentration of $5 \mu \mathrm{M}($ e.g., CS1_341F and CS2_806R; leading to a $250 \mathrm{nM}$ working

depending on experiment) for 20 minutes, followed by two cycles of denaturation for 5 minutes at $95^{\circ} \mathrm{C}$ and annealing at $60^{\circ} \mathrm{C}$ for 20 minutes, and a final extension temperature of $72^{\circ} \mathrm{C}$ for 10 minutes. For temperature gradient experiments, annealing temperatures of $40^{\circ} \mathrm{C}, 45^{\circ} \mathrm{C}, 50^{\circ} \mathrm{C}$, $55^{\circ} \mathrm{C}$, and $60^{\circ} \mathrm{C}$ were tested. For single reverse primer variant (RPV) analyses, an annealing temperature of $50^{\circ} \mathrm{C}$ was used for both TAS and DePCR amplification reactions. Subsequently, a

142 pool composed of $5 \mu \mathrm{l}$ from the first reaction of each sample was collected and processed for 143 cleanup using AMPure XP beads (Beckman-Coulter) at $0.7 \mathrm{X}$ per the manufacturer's recommendations. The cleaning step was performed twice, sequentially. A final elution volume 145 of $20 \mu \mathrm{l}$ was used to concentrate the sample prior to the second stage of the DePCR reaction. The second stage reactions were conducted in a final volume of $20 \mu \mathrm{l}$; the reaction contained $10 \mu 1$ of 
148 (CAAGCAGAAGACGGCATACGA) primers, $2 \mu 1$ of purified template from pooled first stage 149 PCR, and water up to $20 \mu 1$. The thermocycler conditions were: $95^{\circ} \mathrm{C}$ for 3 minutes, 30 cycles at $15095^{\circ} \mathrm{C}$ for 15 seconds, $60^{\circ} \mathrm{C}$ for 30 seconds and $72^{\circ} \mathrm{C}$ for 30 seconds. Prior to sequencing the pool 151 libraries were purified using a Pippin Prep DNA Size Selection System (Sage Science), 152 employing a 2\% agarose gel cassette and selecting for fragment sizes from 450-600 bp.

153 Sequencing of the amplified pool was performed on an Illumina MiSeq employing V2 chemistry 154 and 2x250 base reads, and demultiplexing of sequence data was performed on instrument.

155 Library preparation and sequencing were performed at the UIC Sequencing Core (UICSQC).

Sequence Data Analysis

Raw sequence FASTQ files were merged using the software package PEAR [11], with default parameters. For analysis of primer utilization profiles, merged sequences were trimmed using the software package trimmomatic [12], and sequences shorter than 400 bases and longer than 500 bases were removed. Using Unix bash scripting, exact primer sequences were searched for within these trimmed sequences and counted. For microbial community analysis, PEAR-merged sequences were initially processed through the software package CLC genomics (Qiagen, v10, Aarhus, Denmark) to remove primer sequences, to perform quality trim (below Q20 removed), and size trimming (below 400 bases removed). Sequences were then screened for chimeras using the USEARCH61 algorithm [12], and putative chimeric sequences were removed from the dataset. Subsequently, sequences were pooled and clustered into operational taxonomic units (OTUs) at a threshold of 97\% similarity (QIIME v1.8.0) [13]. Representative sequences from all OTUs were annotated using the UCLUST algorithm and the Greengenes 13_8 reference database [14], and a biological observational matrix (BIOM) was generated by this annotation pipeline 
171 [15]. The BIOM file was analyzed and visualized using the software package Primer7 [16] and

172 the R environment [17]. The R package 'vegan' [18] was employed to generate alpha diversity

173 indices (Shannon, richness, and evenness indices) and to produce rarefied BIOM files. Bray-

174 Curtis dissimilarity indices were calculated within the R package 'vegan' and these indices were

175 used to evaluate differences in composition between samples. Analysis of similarity (ANOSIM)

176 calculations were performed at the taxonomic level of genus, using square root transformed data.

177 Initial analysis and processing of the samples was performed using QIIME (v1.8.0) package

178 scripts. Metric multi-dimensional scaling (mMDS) plots were generated using the cmdscale and

179 ggplot2 functions [19] within the R programming environment. Ellipses, representing a 95\%

180 confidence interval around group centroids, were drawn assuming a multivariate t-distribution.

181 Some visualizations were performed using the software package OriginPro 2018 (OriginLab,

182 Northampton, Mass). Rarefaction and group-significance testing (i.e., non-parametric Kruskal-

183 Wallis test) were performed using the QIIME software package.

Data Archive

Raw sequence data files were submitted in the Sequence Read Archive (SRA) of the

187 National Center for Biotechnology Information (NCBI). The BioProject identifier of the samples 188 is PRJNA506229. Full metadata for each sample are provided in Table S1.

\section{Results}

Theory 
PCR method described previously [4]. We previously noted that the first two cycles of PCR are

194

195

196

197

198

unique in that no amplification of the template is performed. Rather, linear copying of the template nucleic acid prepares the reaction for exponential amplification, starting in the third cycle. In the prior manuscript, linear copying of the original gDNA template was separated from exponential amplification of target copies using exonuclease I (Figure 1B). Locus-specific primers containing 5' linker sequences anneal to genomic DNA during two cycles of amplification. Subsequently, exonuclease I was used to remove unused primers from reaction mixtures. Finally, the copied templates were exponentially amplified using primers targeting the 5 ' linker sequences but not the source genomic DNA. This approach is viable, but cumbersome due to the need for exonuclease treatment of each sample, and for individual amplification of each sample with primers containing Illumina sequencing adapters and sample-specific barcodes.

We modified the original PEX PCR protocol by including both locus-specific primers containing 5' linkers as well as primers with Illumina sequencing adapters, sample-specific barcodes, and 3' linkers together in the first linear stage of the DePCR reaction (Figures 1C and 2). Thus, the DePCR approach combines primer sets used in both stage A and B of the PEX PCR method in the same reaction. In addition, four cycles of linear copying are performed, instead of two as in the PEX PCR method (Figures 1 and 2). The resulting products are target copies containing Illumina sequencing adapter sequences, sample specific barcodes, linker sequences, and the region of interest. The four cycles of copying serve to prepare the templates for exponential amplification but also (unlike PEX PCR) incorporate a sample-specific barcode so that samples can be pooled and amplified exponentially simultaneously in the second stage. As with PEX PCR, 
214 the linear amplification stage - if operating at 100\% efficiency - does not increase the total number

215 of targets from that present in the source template DNA.

216 After linear copying during the first four cycles, the reactions are pooled and purified to remove

217 unincorporated primers. It is essential for the proper functioning of the method that the primers

218 from the initial stage of the reaction are completely removed; otherwise these locus-specific

219 primers continue to interact with template and amplicons during exponential amplification cycles.

220 We observed that a single cleanup using AMPure XP beads (0.7X) was not sufficient to fully

221 remove all primers; therefore, a double cleanup (i.e., two sequential AMPure XP 0.7X cleanups of

222 the pooled reactions) is performed. The final purified DNA includes a range of DNA types, but

223 only the fragments that contain Illumina sequencing adapters at both ends of the molecule have

224 been generated only through linear copying steps and are available for amplification using Illumina

225 P5 and P7 primers (Figure 2). The entire pool is then used as input template for subsequent

226 amplification using primers consisting of Illumina P5 and P7 sequences. Linear-copied DNA

227 fragments from all samples within the pool, each now containing a sample-specific barcode, are

228 thus subject to exponential amplification simultaneously. One useful feature of this approach is

229 that hundreds of samples can be amplified simultaneously within a single reaction. The theoretical

230 advantages of this novel workflow include: (1) the elimination of a separate exonuclease step for

231 each sample, (2) the rapid reduction of many reactions into a single reaction for purification and

232 exponential amplification, and (3) all associated benefits of the prior PEX PCR, in which linear

233 and exponential amplification stages of PCR are isolated from each other and where locus-specific

234 primers are only active for two linear cycles of copying. 
on observed microbial community structure, a single genomic DNA sample was amplified across multiple annealing temperatures using both amplification strategies. Five technical replicates for each condition were performed, and amplicons were sequenced together. The data were analyzed to determine if there were significant differences in sequence metrics (chimera formation), alpha diversity (richness and Shannon index), and observed community structure (beta diversity analyses including multi-dimensional scaling, analysis of similarity (ANOSIM), and taxon-level groupsignificance testing). Rates of detectable chimera formation were several orders of magnitude lower with the DePCR pipeline relative to the TAS pipeline, regardless of annealing temperature (Table 2). Average chimera detection rate for TAS-processed samples range from 5.16 to $6.53 \%$, while that for DePCR-processed samples ranged from $0.03-0.1 \%$; this difference was significant at all annealing temperatures tested (ANOVA, $\mathrm{P}<0.001)$. Low rates of detectable chimeras were found in all experiments conducted with DePCR, with averages in the range of $0.01-0.1 \%$ (Table 2). Conversely, alpha diversity metrics (genus-level richness and Shannon index), were slightly and significantly higher in TAS-based analyses relative to DePCR. Genus-level richness was on average from 1.06-1.21X higher in TAS analyses relative to DePCR, across annealing temperatures from $40^{\circ} \mathrm{C}$ to $60^{\circ} \mathrm{C}$ (one-way ANOVA; $p$ values ranged from $1.9 \mathrm{E}-5$ to $1.3 \mathrm{E}-1$; Table 3). Shannon indices were from 1.03-1.06X higher in TAS analyses relative to DePCR across annealing temperatures from $40^{\circ} \mathrm{C}$ to $60^{\circ} \mathrm{C}$ (ANOVA; $\mathrm{p}<8.13 \mathrm{E}-4$; Table 3). structure was seen in both TAS and DePCR amplification methods (Figure 3A). Although the overall scale of difference between TAS and DePCR was modest (maximum Bray-Curtis 
260 a DePCR sample with $40^{\circ} \mathrm{C}$ ), there was a significant effect of amplification method on observed

261 microbial community at all temperatures (Table S2). Two-way ANOSIM analyses indicated

262 significant differences by temperature across methods ( $\mathrm{R}=0.832 ; \mathrm{p}=0.0001$; Figure 3B), and by

263 amplification method across temperatures $(\mathrm{R}=0.988 ; \mathrm{p}=0.0001$; Figure 3C). Similar trends were

264 observed for increases in annealing temperature in both methods, with temperature loading

265 primarily on MDS axis 1. As previously noted [4], greater variability in observed microbial

266 community structure was noted with DePCR with low annealing temperature, particularly at $40^{\circ} \mathrm{C}$

267 (Figure 3A).

268

269

270

271

One key feature of the DePCR methodology is the ability to determine which primers in a degenerate pool are interacting with the source genomic DNA. This is achieved as the exponential amplification of the template is performed using primers targeting Illumina sequencing adapters and not the locus-specific primers (Figures 1, 2). Locus-specific primers only interact with the gDNA and the first linear copies of gDNA during the first two cycles of the DePCR method. These primer sequences are retained during exponential amplification with primers targeting linker sequences. Conversely, in standard PCR, the locus-specific primers interact with both the genomic DNA template and with copies made from the genomic DNA during exponential amplification; thus, information regarding primer-gDNA template interactions are lost [4]. We thus examined the so-called "primer utilization profiles" (PUPs) for these reactions (Figure 4). The relative frequency of each of the 18 unique primer variants is shown for each replicate at each PCR condition (temperature $\mathrm{x}$ method). Standard PCR amplification protocol (TAS) removes primertemplate interaction information as primer-amplicon interactions throughout the amplification reaction tolerate mismatches; all 18 primer variants are used at similar frequencies, regardless of annealing temperature (Figure 4A). Some patterning is observed in the TAS method, but overall 
283 diversity of primer utilization is extremely high and only small differences were observed between 284 temperatures of $40-60^{\circ} \mathrm{C}$ (Figure 4B). The average Shannon index for PUP profiles of TAS 285 samples across all annealing temperatures was 2.859-2.864; the maximum possible natural log 286 Shannon index for 18 features is 2.890 . This PUP diversity profiling demonstrates that for standard 287 TAS PCR, the primers used in copying throughout the amplification reaction are not dependent on 288 annealing temperature. amplified using the DePCR protocol (Figure 4A, B). A shift in PUP patterning is observed with increasing annealing temperature, and at $60^{\circ} \mathrm{C}$ two primer variants (RPV5 and RPV15) dominate. At lower annealing temperatures, a broader range of primers are utilized in the initial stages of gDNA copying. The relationship between annealing temperature and primer utilization richness (here represented as the Shannon index) was best fit with a polynomial equation and is shown in

Figure 4C. As annealing temperature increases, fewer and fewer primer variants interact with the gDNA template. Conversely, at the lowest tested annealing temperature of $40^{\circ} \mathrm{C}$, the Shannon index of the DePCR amplicons nearly matched that of the TAS. Several primer variants, however, including RPVs 10, 12, 14 and 18, were poorly utilized in DePCR amplifications regardless of annealing temperature (Figure 4A). These four variants included variants with high melting 300 temperatures $\left(57.4,57.5,58\right.$ and $\left.59.8^{\circ} \mathrm{C}\right)$, while the two most utilized RPVs at PCR annealing temperatures of $60^{\circ} \mathrm{C}$ had moderate to high annealing temperatures $\left(56.4\right.$ and $\left.58.7^{\circ} \mathrm{C}\right)$. Thus, the melting temperature of the primer did not directly correlate with utilization at different PCR annealing temperatures in this system. The observed primer utilization profiles represent a template-specific phenomenon, and different PUPs would be recovered with different DNA 305 templates. 

and $\mathrm{P} 7$ primers. The pooling of samples can only be performed because of the incorporation of a sample-specific unique barcode for each sample during the first stage. During the second stage amplification, primers targeting the Illumina adapters are used for amplification, and all templates from all samples are amplified simultaneously (Figure 1C). Since there is no opportunity for primer-template bias during the second stage (i.e., Stage B of Figure 1C) of amplification (all amplifiable template molecules contain Illumina sequencing adapters) and primers are non-degenerate, the relative abundance of template molecules from a single sample within the pool should be maintained during amplification. To determine if the relative abundance of template molecules from each sample was maintained in the DePCR protocol, we performed an experiment in which input gDNA (feces) was varied from $1.25 \mathrm{ng}$ to $20 \mathrm{ng}$ per 10 $\mu 1$ reaction. All input levels were performed with five technical replicates. After the first stage (4 cycles) of the DePCR, all replicates from all gDNA input levels were pooled in equal volume and purified. The purified product was then amplified with P5 and P7 primers, and the final pool sequenced. We first assessed whether the input DNA concentration was correlated with the total number of reads generated using this approach (Figure S1). Since all samples were amplified together, and low input DNA samples should theoretically provide fewer molecules to the combined pool, we hypothesized that a linear relationship should exist between input DNA in the first stage and the number of reads generated per sample. A significant positive correlation between input gDNA concentration and absolute number of reads recovered from each sample 
329 was observed, though substantial variability at each input concentration was observed $\left(\mathrm{R}^{2}=0.58\right.$,

330 Figure S1C). We also sought to determine if the input gDNA concentration from the same

331 sample had a significant effect on the observed microbial community structure. Although there

332 was a positive correlation between input gDNA and total number of sequences recovered, we

333 observed no significant effect of input gDNA on the microbial community structure (Figure

334 S1A; Global ANOSIM R=-0.034; $\mathrm{p}=0.79$ ). Similarly, no significant difference in primer

335 utilization was observed with different gDNA input concentrations (Figure S1B). Thus,

336 increasing input gDNA concentration alters the number of molecules passing to the second stage

337 of the DePCR reaction, but within the observed concentration range does not affect the primer

338 utilization profile or final observed microbial community structure.

Assessing the effect of individual primers in a degenerate primer pool

Degenerate primer pools are generally used to amplify genomic DNA, although not all

primers actively interact with the source gDNA (Figure 4A). This degenerate mixture of primers is employed to target a broad range of taxa, and the presence of additional primer variants in pools has been shown to improve detection of known microbial lineages [20-23]. In standard PCR, all primers do eventually interact with amplified copies of gDNA during the many cycles of exponential amplification; however, many primers do not interact with the source genomic DNA due to preferential annealing of other primers (Figure 4A). We sought, therefore, to determine how much microbial diversity could be detected using each primer variant independently in PCR reactions using both the TAS and DePCR methods. In addition, we sought to determine how the observed microbial community structure differed by single primer variant usage. We hypothesized that the single primer variant PCR would better approximate degenerate 
352 primer pools when using the DePCR method relative to the TAS method, as our prior work

353 showed that a deconstructed PCR approach was more tolerant of mismatches between primer

354 and gDNA template than TAS [4]. The tolerance of mismatches may lead to better capture of

355 microbial community diversity when a greater number of mismatches between primer and

356 template are present, as is expected in a single primer PCR. To explore this, we PCR-amplified a

357 single gDNA template (feces) with the 18 unique reverse primer variants (RPVs) from the

358 degenerate primer pool. Each reaction was performed in technical duplicates, and each reaction

359 was performed using the DePCR and the TAS method. Three RPVs from the TAS method were

360 removed from the analysis due to pipetting error, as determined by primer utilization profiles.

361 These included one replicate of RPV5 and both replicates of RPV15 (Table S1). We compared

362 alpha and beta diversity analyses of the PCRs employing 15-18 unique RPVs to those generated

363 with the fully degenerate primer set. All alpha and beta diversity analyses were performed on

364 data rarefied to a depth of 1800 sequences/sample (Table S1 - experiment 3).

When employing fully degenerate primer pools, observed alpha diversity (Shannon index) of the fecal sample was slightly, but significantly higher when analyzed using the TAS protocol relative to the DePCR protocol (average Shannon index, five replicates, 2.71 to 2.66; ANOVA P $<0.001$; Table 4). We then calculated average Shannon indices for analyses of the same gDNA sample with individual RPVs, employing TAS and DePCR protocols. The average Shannon index for the TAS reactions with unique RPVs (2.40) was significantly lower than that measured for the DePCR reactions (2.58) (ANOVA P<0.001; Table 4). Finally, all RPV data, rarefied to 1800 sequences per sample, was pooled together for TAS and DePCR approaches,

373 independently. These combined datasets were then randomly sub-sampled to 1800 sequences.

374 These rarefactions were performed five times, and the average Shannon index for the combined 
375 RPVs was calculated. In this approach, average Shannon index from TAS (2.48) was

376 significantly lower than for DePCR (2.69) (ANOVA P $<0.001$; Table 4). Across all three

377 methods of calculating observed diversity, there was no significant different in measured

378 Shannon index for the DePCR method (ANOVA, $\mathrm{P}=0.377$ ), while a significant decrease with

379 each RPV independently was observed with the TAS method (ANOVA, P=3.69e-8). When each

380 RPV is used independently in the TAS protocol, the overall captured diversity is lower than with

381 reactions with degenerate pools (Table 4) due to the greater number of potential mismatch

382 interactions that can occur when a complex template is amplified with a single, non-degenerate

383 primer. As the DePCR method is more tolerant of mismatches, no significant decrease in average

384 Shannon index was observed. However, the observed variance in Shannon index among the

385 individual RPVs was greater for the DePCR than for the TAS method (Table 4).

We next examined the structure of the observed fecal microbial communities in standard

387

388

389

390

391

392

393

394

395

396

397

TAS and DePCR with degenerate primer pools, and with reactions conducted using RPVs

(Figure 5). We observed high reproducibility for five replicates using TAS (i.e., 'TAS_pool') or DePCR (i.e., 'DePCR_pool') with degenerate primer pools (Figure 5A, B) and observed microbial community structure was significantly different between TAS and DePCR employing the degenerate primer pools (ANOSIM, $\mathrm{R}=0.401, \mathrm{p}=0.001$ ). Compared to amplifications with degenerate pools of primers, within-group variability was much greater for the analyses of RPVs individually with either amplification protocol (Figure 5A, B, 'TAS' and 'DePCR'). Withingroup Bray-Curtis dissimilarity (BCD) of amplicons from the 15 (TAS) to 18 (DePCR) RPVs ranged from 0.03 to 0.36 for the TAS method and from 0.04 to 0.68 for the DePCR method (ANOVA $\mathrm{P}<0.001$; Figure 5B). Conversely, the within-group BCD for five technical replicates generated with degenerate primer pools were 0.04 to 0.07 for TAS and 0.05 to 0.11 for DePCR 
398 (ANOVA P $<0.001$ ). Profiles of the individual RPVs from DePCR analyses could be divided into

399 two groups: (a) RPVs with profiles highly similar to degenerate primer pool analysis with either

400 DePCR or TAS; and (b) RPVs with profiles divergent from the degenerate pool communities, 401 and more similar to RPVs from TAS amplification reactions. Overall, the observed microbial 402 community structure generated using the DePCR method with RPVs and with degenerate pools

403 was not significantly different (ANOSIM R=-0.306, $\mathrm{p}=0.99$ ). Conversely, the observed microbial 404 community structure generated using RPVs was significantly different that that observed with 405 degenerate primer pools for the TAS method (ANOSIM $\mathrm{R}=0.487 ; \mathrm{p}=0.003$ ). Average BCD 406 between TAS_pool and TAS RPV profiles (0.211) was significantly greater than for DePCR_pool and DePCR RPV (0.154) (ANOVA P $<0.001$; Figure 5C). DePCR BCD profiles

408 were heavily weighted toward low dissimilarity, with a long tail of high dissimilarity 409 comparisons. The long tail is a result of some primers generating highly divergent observed microbial communities with the DePCR protocol. Many of the primers which showed the poorest utilization within the degenerate pool (e.g., RPV10, 12, 14, and 18; node with red dot in Figure 4A), generated the most divergent single RPV profiles. This suggests that these primers do not 413 closely match the most dominant taxa within this particular gDNA sample.

\section{Discussion}

We demonstrate here an updated protocol for the Deconstructed PCR methodology [4] which reduces the overall complexity of the workflow and increases the throughput. Complete 418 removal of $1^{\text {st }}$ stage (or "Stage A") primers (locus-specific primers containing 5' overhanging linkers) is essential for the effectiveness of the DePCR protocol, and we have replaced the exonuclease step with a bead-based magnetic cleanup. The new method improves throughput by 
421 generating barcoded DNA fragments through 4 cycles of linear amplification; thus, all samples

422 can be pooled before bead-cleanup. This reduces workflow complexity and cost, while retaining

423 the essential features of the DePCR reaction. Complete removal of primers is difficult to measure

424 directly, however; thus, the primer utilization profiles (PUPs) are the clearest indication of

425 successful removal of locus-specific degenerate primers from the first stage of the reaction. With

426 standard PCR, no true signal is obtained from the PUPs, as primer-amplicon interactions during

427 late cycles generates a 'scrambled' signal due to mismatch interaction with amplicons present at

428 high abundance. In DePCR, a PUP signal can be obtained as locus-specific primers only interact

429 with the gDNA template and linear copies during the first two cycles of PCR. Subsequently, all

430 exponential amplification is performed using conserved sequences that are not present in the

431 source gDNA. In this way, the primer sequences interacting with the source gDNA are

432 'fossilized' and can be interrogated directly. When using this approach, we observed strong

433 effects of annealing temperature on primer-gDNA template interactions, with a negative

434 quadratic correlation between annealing temperature and evenness of primer utilization. At

435 highest annealing temperatures, very few primers from the primer pool anneal to the gDNA

436 template, and this leads to a shift in the sequences that are amplified by PCR with a result of

437 significantly different observed microbial communities. We note that the elevated annealing

438 temperature by itself does not select for primer variants with the highest theoretical melting

439 temperature. Rather, primer variants, presumably template-specific, are favored regardless of

440 their melting temperature.

441 A surprising benefit to the DePCR methodology is the reduced rate of chimera

442 formation. Chimeras are artifactual hybrid sequences generated from two or more templates due

443 to incomplete polymerase extension during PCR, and their presence can be difficult to detect and 
444 lead to overestimation of diversity and alteration of observed microbial community structure [24-

445 26]. Input genomic DNA concentration and target microbial community complexity have been

446 identified as contributors $[27,28]$. We previously observed that chimera formation was

447 correlated with total number of PCR cycles in both first and second stages of PCR [29], and this

448 has been reported elsewhere in many studies [26, 28, 30]. As many factors can contribute to

449 chimera formation, various solutions have been proposed, including reducing input gDNA

450 concentration [31], reducing PCR cycles [32, 33], employing highly processive enzymes [28],

451 among others. In this study, we have observed that the use of the DePCR methodology can

452 dramatically and significantly lower rates of observed chimeras resulting in rates that were

453 generally below $0.1 \%$. These low rates of chimera formation were observed across all annealing

454 temperatures and input template concentrations tested. The reasons for the dramatic decrease in

455 chimera formation rate with the DePCR method are likely a result of: (a) reduction in input DNA

456 concentration for exponential amplification due to the double-purification step, (b) higher

457 annealing temperature for the exponential amplification due to targeting of P5/P7 Illumina

458 adapters -potentially reducing the re-annealing of PCR products to other products, and (c) long

459 elongation times during the first cycles, reducing the formation of incomplete molecules during

460 the first stages of PCR. Conceivably, chimera formation with DePCR could be reduced further;

461 we performed 30 cycles of amplification to generate robust PCR yields for sequencing.

462 However, the amplification of the pool of amplicons during the second stage PCR could be

463 titrated across different numbers of cycles, and the reaction with the fewest numbers of cycles

464 yielding sufficient DNA for sequencing could be employed. It is critical to remember that the

465 rate of chimera formation represents only the rate of detectable chimera formation, and that

466 chimeras generated from closely related sequences are not only likely to occur at higher rates 
467 [30] but are also essentially undetectable by chimera detection software. We note that in this

468 study, amplification of fecal gDNA with degenerate primer pools resulted in higher observed

469 diversity with the TAS method relative to the same sample amplified with the DePCR protocol

470 (Table 4), and this could represent the residual presence of chimeras that were not removed.

Suzuki and Giovannoni [32] previously modeled PCR reactions with mixed templates by

472 incorporating efficiency parameters into equations estimating molarity of amplicon yield. They

473 further estimated second-order kinetics wherein changes in the concentration of specific PCR

474 products alter efficiencies during the amplification, including through inhibition of amplification

475 by competition between primers and amplicons for annealing locations. With increasing cycle

476 number, reaction efficiency dropped dramatically. The DePCR method theoretically circumvents

477 at least some of these issues. First, since locus-specific primers interact with template only

478 during two cycles of copying (linear only), any differences in amplification efficiency of

479 templates are limited to those two cycles. Subsequently, all templates are amplified with primers

480 targeting sequences common to all amplifiable templates. Suzuki and Giovannoni [32] showed

481 that even a relatively high amplification efficiency could lead to dramatic distortion of the

482 underlying template ratios within 10-15 cycles. In DePCR approaches, amplification efficiency

483 is expected to be lowest during the first two cycles - when primers anneal to gDNA templates

484 with varying numbers of mismatches - and then higher during the remaining cycles as

485 amplification is performed with perfectly matching primers. We also note that in PCRs with

486 degenerate primers, each primer variant is present at a low concentration (total primer

487 concentration / number of variants); in the $2^{\text {nd }}$ stage of the DePCR protocol, a non-degenerate

488 primer at a high concentration relative to each variant is used for amplification. Thus, DePCR

489 limits the number of cycles operating at low primer efficiency and uses high-efficiency reactions 
490 to perform exponential amplification. Degenerate locus-specific primer interactions with PCR

491 amplicons are also removed, thereby removing additional variable efficiency annealing steps

492 from the PCR.

493

We previously demonstrated that a deconstructed PCR approach could help overcome

494 PCR distortions due to mismatches between primers and templates in a mock community [4],

495 and we believe this is in part due to the circumventing of multiple cycles with low amplification

496 efficiency. Single mismatches between templates and primers can substantially alter observed

497 microbial community structure, and indeed, many modifications to degenerate primer pools are

498 performed to increase degeneracy by adding single variants targeting specific microbial taxa

499 [25]. In this study, we independently used each primer variant in a degenerate primer pool both

500 to examine the potential for each primer to amplify a complex microbial gDNA template and to

501 assess the ability of the DePCR protocol to enable single non-degenerate primers to broadly

502 amplify microbial taxa with mismatches. We observed that while the observed microbial

503 community structure varied widely using non-degenerate primer variants (both TAS and

504 DePCR), many single non-degenerate primer variants were able to generate reasonable

505 approximations of the microbial community structure as revealed through amplification reactions

506 with degenerate primer pools, thus indicating that the DePCR approach can be used with

507 complex microbial samples to improve tolerance of mismatches. This suggests that a more

508 empirical approach to primer design can be taken by using the DePCR method to reduce the

509 complexity of degenerate primer pools or enable broader target range of highly degenerate

510 primer pools targeting functional genes. Primer utilization profiling can in turn be used to

511 provide empirical evidence demonstrating which primers within the degenerate primer pool are

512 interacting with unknown templates. The inclusion of non-essential variants decreases the 
513 concentration of all other primers in a primer pool, and removal of unneeded primer variants may

514 be beneficial. However, when using the same primer set for a broad range of complex genomic

515 DNA samples from different environments, we expect that the 'essential' primers will vary from

516 system to system.

517 We can recommend the DePCR protocol for reactions where degenerate primer pools are

518 used or for primer-template systems where mismatches are possible or expected. Several caveats,

519 however, should be considered. First, the method is not recommended for reactions requiring

520 stringent PCR conditions. Second, since reactions are pooled together after the first linear cycles

521 and then amplified, the reactions are sensitive to the relative number of copies within each

522 sample. As observed in Figure S1C, there is a linear response between input gDNA and number

523 of sequences generated. Thus, input gDNA concentration of similar samples should be carefully

524 controlled to avoid large variance in number of sequences generated per sample. Furthermore,

525 different sample types should be amplified independently, as different sample types may have a

526 different density of targets per ng of DNA, leading to further variance in sequence reads

527 generated. Samples with low input DNA concentrations may require additional cycles of

528 exponential amplification during the second stage of DePCR to generate sufficient product for

529 sequencing. Such samples could be processed independently and then pooled with other sample

530 types prior to sequencing. Third, in the updated DePCR protocol where Illumina P5 and P7

531 primer are used, polymerase extension copies through the DNA region containing the sample-

532 specific barcode and can introduce errors. In this study, we employed Fluidigm Access Array

533 primers which contain 10-base barcodes with a Hamming distance of 3 (each barcode has at least

5343 mismatches with all other barcodes), and this large Hamming distance should limit mis-

535 assignment of reads. However, with other barcoding systems, or with very high PCR cycle or 
536 error-prone polymerases, this source of error could lead to cross-signaling between samples or

537 loss of reads. Finally, we note that when assessing if a DePCR protocol is functioning properly,

538 it is important to employ an analysis of primer utilization across a temperature gradient analysis

539 with standard (TAS) and DePCR workflows. In standard PCR, a small or no effect of

540 temperature should be observed on the PUPs, while a strong shift in primer utilization should be

541 observed with the DePCR protocol. Since primer utilization with DePCR can be extremely broad

542 at low annealing temperatures, it can be difficult to differentiate between a properly operating or

543 failed DePCR protocol without the temperature gradient analysis.

\section{Conclusions}

545 We demonstrate here an improved method to reduce bias associated with PCR

546 amplification of complex genomic DNA templates with degenerate primers. The method,

547 DePCR, is a simple and versatile, two-stage PCR protocol allowing highly multiplexed library

548 preparation for Illumina sequencers. When employing this method using a common degenerate

549 primer set targeting microbial 16S rRNA genes, we observed a significant decrease in chimera

550 formation relative to standard PCR amplification. When using non-degenerate primers, the

551 DePCR methodology frequently reduced PCR distortions due to mismatches between primers

552 and templates.

553

554 Figure Legends

555 Figure 1. Schematic of (A) standard (TAS), (B) polymerase-exonuclease (PEX) PCR, and

556 (C) Deconstructed PCR (DePCR) workflows. AT = annealing temperature; ET = Elongation 
557 time. $\mathrm{CS} 1=$ common sequence 1 adapter. $\mathrm{CS} 2=$ common sequence 2 adapter. $\mathrm{BC}=$ barcode. FP

$558=$ Forward primer. RP $=$ Reverse primer. Primer sequences are shown in Figure 2 and Table 1.

559 Figure 2. Polymerase-generated intermediates in the first stage ("Stage A") of the DePCR

560 workflow. Polymerase-extension products generated during the first four cycles of the first stage

561 of the DePCR are shown. After four linear cycles of copying, the first stage is terminated,

562 samples are pooled and purified, and subsequently amplified with Illumina adapter primers.

563 Primers used in this study are shown at the top of the figure, with different functional regions

564 color coded. Red regions represent locus-specific portions of primers. Blue regions represent

565 linker portions of primers. Yellow regions represent Illumina adapter sequences. Purple regions

566 represent a variable, sample-specific barcode. Dotted lines represent nucleotide incorporation by

567 DNA polymerase.

568 Figure 3. Effect of PCR methodology and annealing temperature on observed microbial

569 communities. Genus-level abundance data were visualized using metric MDS (mMDS)

570 ordination employing a distance matrix based on Bray-Curtis similarity. For each PCR condition

571 (TAS or DePCR), five technical replicates were analyzed using annealing temperatures of $40^{\circ}$,

$57245^{\circ}, 50^{\circ}, 55^{\circ}$ or $60^{\circ}$ Celsius. Ellipses represent $95 \%$ confidence intervals around centroids.

573 Rarefaction was performed to a depth of 4,500 sequences per sample. Observed community

574 structure was significantly different across (A) all combinations of temperature and method (one-

575 way ANOSIM Global $\mathrm{R}=0.713 ; \mathrm{P}=0.0001$ ); (B) temperature (two-way ANOSIM R=0.832;

$576 \mathrm{p}=0.0001$ ), and $(\mathbf{C})$ amplification method (two-way ANOSIM R=0.988; $\mathrm{P}=0.0001$ ).

577 Figure 4. Effect of annealing temperature and amplification methodology on primer

578 utilization profiles (PUPs). (A) Two-way clustered heatmap of log-transformed primer variant

579 utilization during amplification of fecal genomic DNA. Samples (columns) are color-coded by 
580 amplification method (TAS or DePCR) and amplification annealing temperature $\left(40^{\circ}, 45^{\circ}, 50^{\circ}\right.$,

$58155^{\circ}$ and $60^{\circ} \mathrm{C}$ ), with five technical replicates per condition and rarefaction to 1,800

582 sequences/sample. Primers (rows) are clustered by profile similarity across all samples and

583 represent all 18 primer variants (RPV1 - RPV18) present in the 806R degenerate primer pool.

584 Theoretical melting temperatures for each primer are shown adjacent to primer name. (B) mMDS

585 ordination of PUPs based on Bray-Curtis similarity. Vectors represent Pearson correlations

$586(>0.9)$ for each primer variant. Ellipses represent 95\% confidence intervals around centroids for

587 DePCR amplification reactions. Five technical replicates per condition were generated and for

588 each sample, rarefaction was performed to 1,800 sequences. (C) Regression analysis was

589 performed was performed on average Shannon index values for primer utilization for each

590 methodology (TAS and DePCR) across annealing temperature. A very small effect of annealing

591 temperature on primer utilization evenness was observed in TAS (orange line). A negative

592 quadratic relationship was observed between annealing temperature and primer utilization

593 evenness in DePCR (blue line). Analyses were based on five technical replicates rarefied to

5944,500 sequences per sample.

595 Figure 5. Microbial community structure revealed using individual primer variants with

596 TAS and DePCR amplification methodologies. (A) Fecal gDNA was amplified using the 341F

597 primer with 18 unique 806R reverse primer variants (RPVs) under standard PCR (TAS) and

598 DePCR workflows. Three RPVs were removed from the TAS analysis due to pipetting error, as

599 described in the text. Genus-level biological observation matrices (BIOMs) were visualized

600 using mMDS. Each amplification with a unique RPV was performed in technical duplicate, and

601 five technical replicates were generated using degenerate primer pools (TAS_pool or

602 DePCR_pool). All samples were rarefied to 1,800 sequences. Ellipses represent 95\% confidence 
603 intervals around centroids. TAS profiles generated with RPVs were significantly distinct from 604 TAS profiles generated with degenerate primer pools (ANOSIM R=0.487; $\mathrm{P}=0.003$ ). DePCR 605 profiles generated with RPVs were not significantly distinct from DePCR profiles generated with 606 degenerate primer pools (ANOSIM R=-0.306; $\mathrm{P}=0.99$ ). (B) Within-group Bray-Curtis 607 dissimilarity distributions for profiles generated with RPVs and with degenerate pools. (C) 608 Between-group Bray-Curtis dissimilarity distributions for observed microbial community 609 structure generated with RPVs and with degenerate primer pools. Average dissimilarity among 610 TAS_pool and TAS RPV profiles (0.211) was greater than for DePCR_pool and DePCR RPV 611 profiles (0.154) (ANOVA $\mathrm{P}<0.001)$.

612 Figure S1. Effect of input gDNA template concentration on microbial community 613 composition and PUPs using DePCR. Analyses were performed on rarefied data sets $(8,000$ 614 sequences per sample), with five technical replicates for each DNA input level $(1.25,2.5,5,10$ 615 or $20 \mathrm{ng} / \mu \mathrm{l}$ ). (A) Genus-level mMDS ordination of microbial community structure using a 616 distance matrix based on Bray-Curtis similarity. No significant differences were observed 617 between all the concentrations (Global ANOSIM: $\mathrm{R}=-0.03376, \mathrm{p}=0.79$ ). Ellipses represent a 95\% 618 confidence interval around the centroid. (B) Primer utilization profiles for all primer variants 619 (RPV1 - RPV18), visualized as a heatmap. (C) A positive correlation between input gDNA $620(1.25,2.5,5,10,20 \mathrm{ng} / \mu \mathrm{l})$ and sequence yield was observed. For all input levels, the same gDNA template was used with five technical replicates. All samples were pooled after stage A of

622 DePCR and amplified together using Illumina P5 and P7 primers. Data were rarefied to 8,000 623 sequences per sample. 
626 Table 1. Primers used in this study. Locus-specific primers were synthesized with linker

627 sequences to allow for two-stage PCR amplification and incorporation of sample-specific

628 barcodes, as described in the text. Primer 806R is 18 -fold degenerate, and variants were

629 synthesized as a pool as well as individually. Access Array primer sequences, synthesized by

630 Fluidigm (PE1-CS1 and PE2-[BC]-CS2), are shown in Figure 2.

631 Table 2. Rates of detectable chimeras in sequence data. Average rates of detectable chimeras

632 are shown for each experiment performed in this study. Significantly lower rates of chimera

633 formation were observed for DePCR-amplified gDNA samples relative to TAS-amplified

634 samples, across multiple annealing temperatures. No significant difference in chimera formation

635 was observed with DePCR methodology with varying gDNA input levels. Significantly higher

636 chimera formation was also observed with TAS relative to DePCR when individual primer

637 variants (RPVs) were utilized. $\mathrm{SD}=$ standard deviation.

Table 3. Alpha diversity indices of observed microbial communities. Shannon indices were

calculated at the taxonomic levels of genus for all samples amplified using TAS and DePCR

640

methodologies across five annealing temperatures of $40^{\circ}, 45^{\circ}, 50^{\circ}, 55^{\circ}$ and $60^{\circ} \mathrm{C}$. Datasets were

641

rarefied to 4,500 sequences/sample. For each methodology and annealing temperature, an

642

average and standard deviation of five technical replicates is shown. At all temperatures, TAS-

643

amplified samples had higher Shannon indices relative to DePCR-amplified samples. SD =

644 standard deviation.

Table 4. Effects of amplification method and reverse primer variants on observed

microbial community alpha diversity. Fecal gDNA was amplified by PCR with 18-fold

647 degenerate reverse primer pools ( 5 technical replicates), and with each unique reverse primer

648 variant (RPV; 2 technical replicates). Data sets were rarefied to 1,800 sequences per sample, and 
649 Shannon indices (loge) were calculated. When using fully degenerate primer pools, average

650 Shannon index was significantly higher for TAS methodology relative to DePCR methodology.

651 When data from all reactions with individual RPVs were analyzed, average Shannon index was

652 significantly lower for TAS methodology relative to DePCR methodology. Data from RPVs

653 (1,800 sequences/sample) were pooled and re-rarefied to 1,800 sequences ( 5 repetitions), and the

654 resulting average Shannon index was significantly lower for the TAS methodology relative to

655 DePCR methodology. Different approaches with the DePCR method did not generate

656 significantly different Shannon indices (ANOVA P=0.377), while the same approaches

657 generated significantly different Shannon indices (ANOVA $\mathrm{P}<0.001$ ).

658 Table S1: Mapping file metadata associated with all samples used in this study. TAS =

659 Targeted amplicon sequencing (standard PCR method). For some samples, multiple rarefactions

660 depths were employed performing different comparisons.

661

662

663

664

665

666

667

668

669

670

671

\section{Table S2: Effect of PCR methodology on observed microbial relative abundance across}

annealing temperatures. Group-significance testing was performed on rarefied biological observation matrices (BIOMs) as described in the text. Non-parametric Kruskal-Wallis testing was performed on taxa greater than $1 \%$ of the total dataset, and p-values, false-discovery rate (FDR) p-values, and Bonferroni-Hochberg corrected p-values are reported. Analyses were performed at taxonomic levels from phylum to species. (A) Comparison of TAS and DePCR across all annealing temperatures simultaneously; data were rarefied to 4,500 sequences/sample; (B) Comparison of TAS and DePCR at an annealing temperature of $40^{\circ} \mathrm{C}$; data were rarefied to 4,500 sequences/sample; (C) Comparison of TAS and DePCR at an annealing temperature of $45^{\circ} \mathrm{C}$; data were rarefied to 16,000 sequences/sample; (D) Comparison of TAS and DePCR at an annealing temperature of $50^{\circ} \mathrm{C}$; data were rarefied to 34,000 sequences/sample; (E) Comparison 
672 of TAS and DePCR at an annealing temperature of $55^{\circ} \mathrm{C}$; data were rarefied to 34,000

673 sequences/sample; (F) Comparison of TAS and DePCR at an annealing temperature of $60^{\circ} \mathrm{C}$;

674 data were rarefied to 16,000 sequences/sample.

1. Caporaso JG, Lauber CL, Walters WA, Berg-Lyons D, Huntley J, Fierer N, Owens SM, Betley J, Fraser $\mathrm{L}$, Bauer M: Ultra-high-throughput microbial community analysis on the Illumina HiSeq and MiSeq platforms. The ISME journal 2012, 6(8):1621.

2. Klappenbach JA, Saxman PR, Cole JR, Schmidt TM: rrndb: the ribosomal RNA operon copy number database. Nucleic acids research 2001, 29(1):181-184.

3. Angly FE, Dennis PG, Skarshewski A, Vanwonterghem I, Hugenholtz P, Tyson GW: CopyRighter: a rapid tool for improving the accuracy of microbial community profiles through lineage-specific gene copy number correction. Microbiome 2014, 2(1):11.

4. Green SJ, Venkatramanan R, Naqib A: Deconstructing the polymerase chain reaction: understanding and correcting bias associated with primer degeneracies and primer-template mismatches. PloS one 2015, 10(5):e0128122.

5. Bohlander SK, Espinosa III R, Le Beau MM, Rowley JD, Díaz MO: A method for the rapid sequence-independent amplification of microdissected chromosomal material. Genomics 1992, 13(4):1322-1324.

6. Muyzer G, De Waal EC, Uitterlinden AG: Profiling of complex microbial populations by denaturing gradient gel electrophoresis analysis of polymerase chain reaction-amplified genes coding for 16S rRNA. Applied environmental microbiology 1993, 59(3):695-700.

7. Caporaso JG, Lauber CL, Walters WA, Berg-Lyons D, Lozupone CA, Turnbaugh PJ, Fierer N, Knight R: Global patterns of 16S rRNA diversity at a depth of millions of sequences per sample. Proceedings of the national academy of sciences 2011, 108(Supplement 1):4516-4522.

8. Walters W, Hyde ER, Berg-Lyons D, Ackermann G, Humphrey G, Parada A, Gilbert JA, Jansson JK, Caporaso JG, Fuhrman JA: Improved bacterial 16S rRNA gene (V4 and V4-5) and fungal internal transcribed spacer marker gene primers for microbial community surveys. Msystems 2016, 1(1):e00009-00015.

9. Owczarzy R, Tataurov AV, Wu Y, Manthey JA, McQuisten KA, Almabrazi HG, Pedersen KF, Lin Y, Garretson J, McEntaggart NO: IDT SciTools: a suite for analysis and design of nucleic acid oligomers. Nucleic acids research 2008, 36(suppl_2):W163-W169.

10. Naqib A, Poggi S, Wang W, Hyde M, Kunstman K, Green SJ: Making and Sequencing Heavily Multiplexed, High-Throughput 16S Ribosomal RNA Gene Amplicon Libraries Using a Flexible, Two-Stage PCR Protocol. In: Gene Expression Analysis. Springer; 2018: 149-169.

11. Zhang J, Kobert K, Flouri T, Stamatakis A: PEAR: a fast and accurate Illumina Paired-End reAd mergeR. Bioinformatics 2013, 30(5):614-620.

12. Edgar R: Usearch. In.: Lawrence Berkeley National Laboratory (LBNL), Berkeley, CA (United States); 2010. 
711 13. Caporaso JG, Kuczynski J, Stombaugh J, Bittinger K, Bushman FD, Costello EK, Fierer N, Pena AG,

712

713

714

715

716

717

718

719

720

721

722

723

724

725

726

727

728

729

730

731

732

733

734

735

736

737

738

739

740

741

742

743

744

745

746

747

748

749

750

751

752

753

754

755

Goodrich JK, Gordon JI: QIIME allows analysis of high-throughput community sequencing data. Nature methods 2010, 7(5):335.

14. McDonald D, Price MN, Goodrich J, Nawrocki EP, DeSantis TZ, Probst A, Andersen GL, Knight R, Hugenholtz P: An improved Greengenes taxonomy with explicit ranks for ecological and evolutionary analyses of bacteria and archaea. The ISME journal 2012, 6(3):610.

15. McDonald D, Clemente JC, Kuczynski J, Rideout JR, Stombaugh J, Wendel D, Wilke A, Huse S, Hufnagle J, Meyer F: The Biological Observation Matrix (BIOM) format or: how I learned to stop worrying and love the ome-ome. GigaScience 2012, 1(1):7.

16. Clarke K, Gorley R: Getting started with PRIMER v7. PRIMER-E: Plymouth, Plymouth Marine Laboratory 2015.

17. Team RC: R: A language and environment for statistical computing. 2013.

18. Oksanen J, Blanchet FG, Kindt R, Legendre P, Minchin PR, O'hara R, Simpson GL, Solymos P, Stevens $\mathrm{MHH}$, Wagner $\mathrm{H}$ : vegan: Community ecology package. $R$ package version 2011:117118.

19. Wickham HJJSS: ggplot2: elegant graphics for data analysis. 2010, 35(1):65-88.

20. Hayashi $\mathrm{H}$, Sakamoto $\mathrm{M}$, Benno $\mathrm{Y}$ : Evaluation of three different forward primers by terminal restriction fragment length polymorphism analysis for determination of fecal Bifidobacterium spp. in healthy subjects. Microbiology and immunology

2004, 48(1):1-6.

21. Frank JA, Reich Cl, Sharma S, Weisbaum JS, Wilson BA, Olsen GJ: Critical evaluation of two primers commonly used for amplification of bacterial 16S rRNA genes. Applied environmental microbiology 2008, 74(8):2461-2470.

22. Parada AE, Needham DM, Fuhrman JA: Every base matters: assessing small subunit rRNA primers for marine microbiomes with mock communities, time series and global field samples. Environmental microbiology 2016, 18(5):1403-1414.

23. Apprill A, McNally S, Parsons R, Weber L: Minor revision to V4 region SSU rRNA 806R gene primer greatly increases detection of SAR11 bacterioplankton. Aquatic Microbial Ecology 2015, 75(2):129-137.

24. Hugenholtz $\mathrm{P}$, Huber T: Chimeric 16S rDNA sequences of diverse origin are accumulating in the public databases. International journal of systematic and evolutionary microbiology 2003, 53(1):289-293.

25. Schloss PD, Gevers D, Westcott SL: Reducing the effects of PCR amplification and sequencing artifacts on 165 rRNA-based studies. PloS one 2011, 6(12):e27310.

26. Edgar RC, Haas BJ, Clemente JC, Quince C, Knight R: UCHIME improves sensitivity and speed of chimera detection. Bioinformatics 2011, 27(16):2194-2200.

27. Fonseca V, Nichols B, Lallias D, Quince C, Carvalho G, Power D, Creer S: Sample richness and genetic diversity as drivers of chimera formation in nSSU metagenetic analyses. Nucleic Acids Research 2012, 40(9):e66-e66.

28. Lahr DJ, Katz LA: Reducing the impact of PCR-mediated recombination in molecular evolution and environmental studies using a new-generation high-fidelity DNA polymerase. Biotechniques 2009, 47(4):857-866.

29. Ionescu D, Overholt WA, Lynch MD, Neufeld JD, Naqib A, Green SJ: Microbial community analysis using high-throughput amplicon sequencing. In: Manual of Environmental Microbiology, Fourth Edition. American Society of Microbiology; 2016: 2.4. 2-1-2.4. 2-26.

Peer) reviewing PDF | (2019:02:34879:2:0:NEW 30 Apr 2019) 
756 30. Wang GC, Wang Y: The frequency of chimeric molecules as a consequence of PCR coamplification of 16S rRNA genes from different bacterial species. Microbiology 1996,

759 142(5):1107-1114.

760

31. D'Amore R, Ijaz UZ, Schirmer M, Kenny JG, Gregory R, Darby AC, Shakya M, Podar M, Quince C, Hall N: A comprehensive benchmarking study of protocols and sequencing platforms for $16 \mathrm{~S}$

761

762

763

764

765 rRNA community profiling. BMC genomics 2016, 17(1):55.

32. Suzuki MT, Giovannoni SJ: Bias caused by template annealing in the amplification of mixtures of 16S rRNA genes by PCR. Applied environmental microbiology 1996, 62(2):625-630.

33. Kanagawa T: Bias and artifacts in multitemplate polymerase chain reactions (PCR). Journal of bioscience and bioengineering 2003, 96(4):317-323. 
Figure 1

Schematic of (A) standard (TAS), (B) polymerase-exonuclease (PEX) PCR, and (C) Deconstructed PCR (DePCR) workflows.

$\mathrm{AT}=$ annealing temperature; $\mathrm{ET}=$ Elongation time. $\mathrm{CS} 1=$ common sequence 1 adapter. $\mathrm{CS} 2$ $=$ common sequence 2 adapter. $\mathrm{BC}=$ barcode. $\mathrm{FP}=$ Forward primer. $\mathrm{RP}=$ Reverse primer. Primer sequences are shown in Figure $\mathbf{2}$ and Table $\mathbf{1 .}$
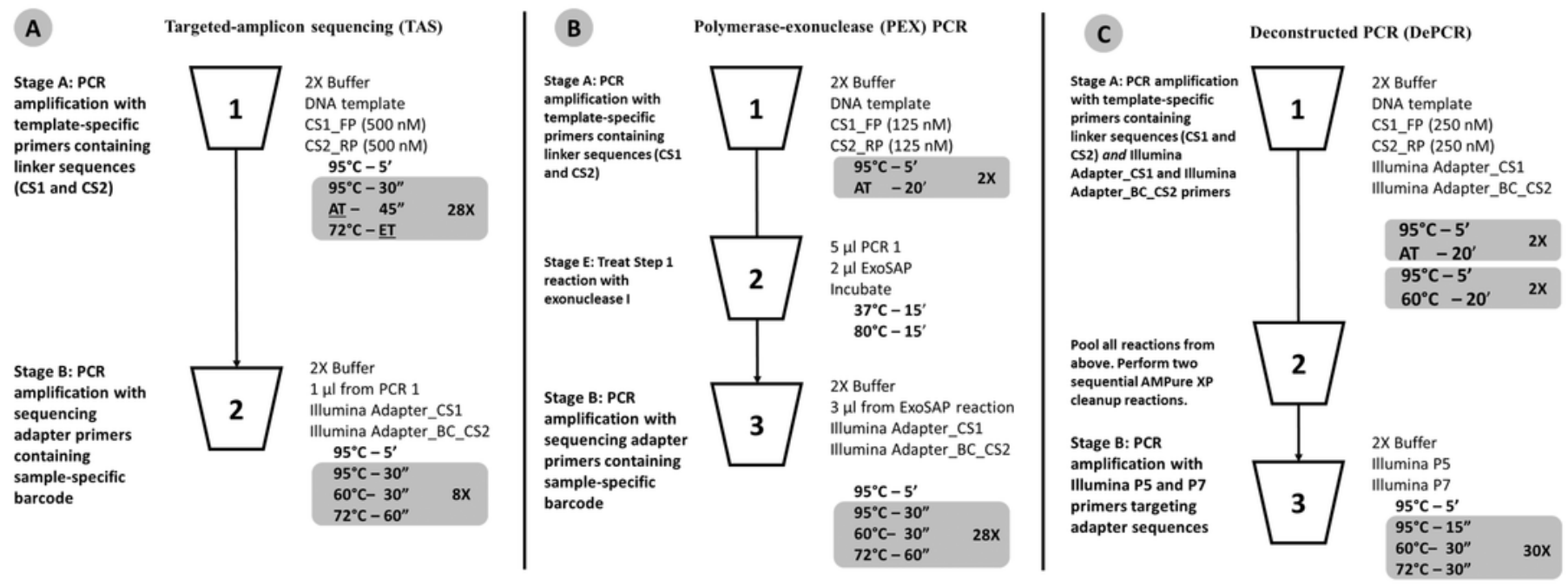


\section{Figure 2 (on next page)}

Polymerase-generated intermediates in the first stage ("Stage A") of the DePCR workflow.

Polymerase-extension products generated during the first four cycles of the first stage of the DePCR are shown. After four linear cycles of copying, the first stage is terminated, samples are pooled and purified, and subsequently amplified with Illumina adapter primers. Primers used in this study are shown at the top of the figure, with different functional regions color coded. Red regions represent locus-specific portions of primers. Blue regions represent linker portions of primers. Yellow regions represent Illumina adapter sequences. Purple regions represent a variable, sample-specific barcode. Dotted lines represent nucleotide incorporation by DNA polymerase. 


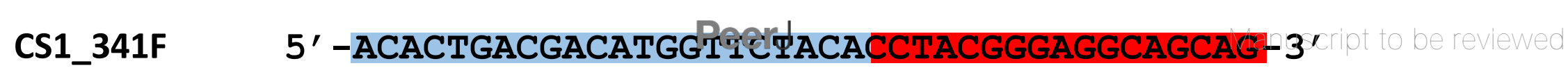

CS2_806R 5' -TACGGTAGCAGAGACTTGGTCTGGACTACHVGGGTWTCTAAT-3'

PE1-CS1 5' -AATGATACGGCGACCACCGAGATCTACACTGACGACATGGTTCTACA-3'

PE2-[BC]-CS2 5' -CAAGCAGAAGACGGCATACGAGATXXXXXXXXXXTACGGTAGCAGAGACTTGGTCT-3'

P5 5' -AATGATACGGCGACCACCGA- $3^{\prime}$

P7 5' -CAAGCAGAAGACGGCATACGA-3'

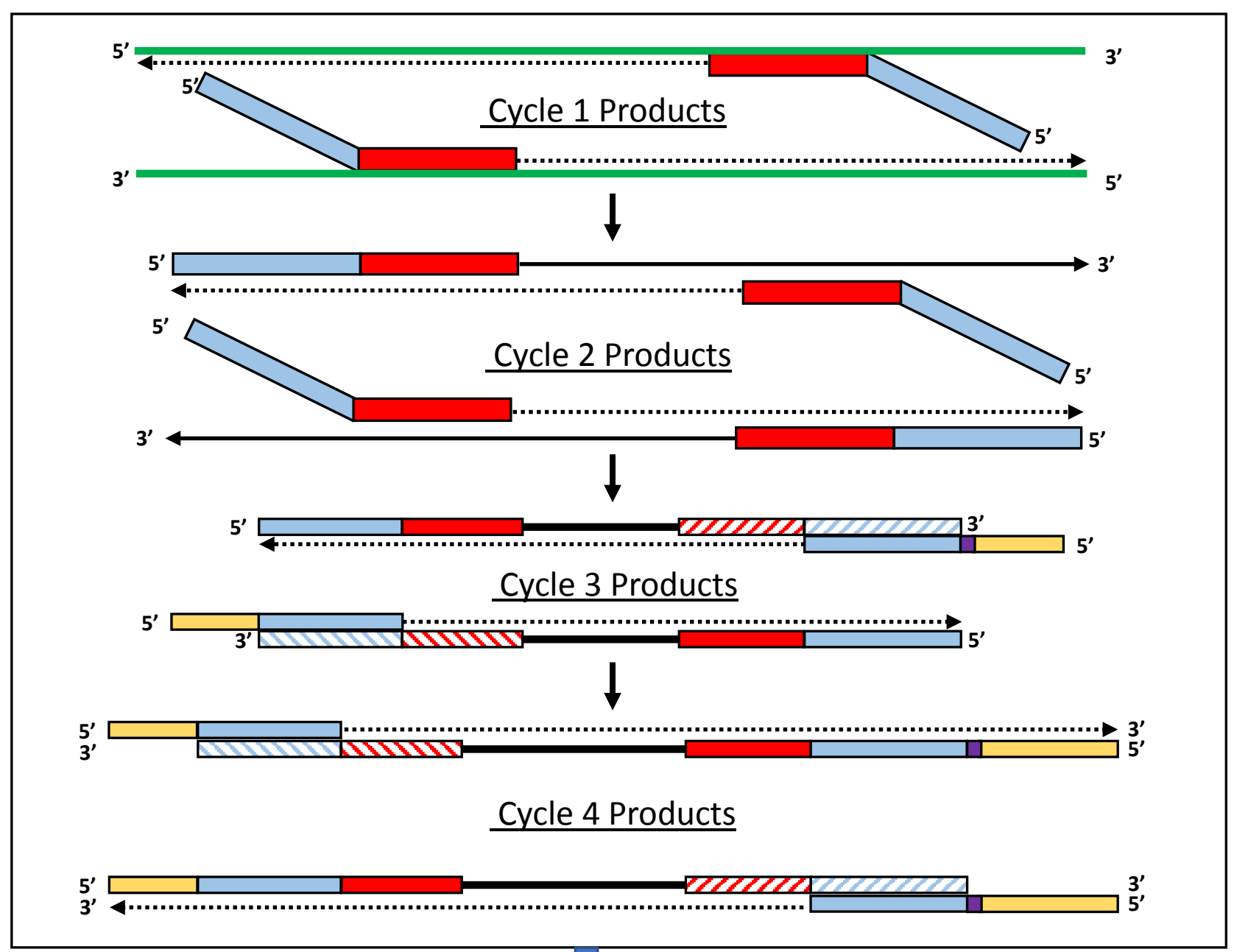

Linker sequences [CS1 and CS2 linkers shown]

Locus-specific primer

Sample-specific barcode

Sequencing adapters

Template DNA

Copied DNA from prior cycle

Copied DNA generated during current cycle

NIIINI Inverse complement 


\section{Figure 3 (on next page)}

Effect of PCR methodology and annealing temperature on observed microbial communities.

Genus-level abundance data were visualized using metric MDS (mMDS) ordination employing a distance matrix based on Bray-Curtis similarity. For each PCR condition (TAS or DePCR), five technical replicates were analyzed using annealing temperatures of $40^{\circ}, 45^{\circ}, 50^{\circ}, 55^{\circ}$ or $60^{\circ}$ Celsius. Ellipses represent $95 \%$ confidence intervals around centroids. Rarefaction was performed to a depth of 4,500 sequences per sample. Observed community structure was significantly different across (A) all combinations of temperature and method (one-way ANOSIM Global $R=0.713 ; P=0.0001$ ); (B) temperature (two-way ANOSIM $R=0.832$;

$\mathrm{p}=0.0001$ ), and $(\mathbf{C})$ amplification method (two-way ANOSIM $\mathrm{R}=0.988 ; \mathrm{P}=0.0001$ ). 


\section{Figure 4 (on next page)}

Effect of annealing temperature and amplification methodology on primer utilization profiles (PUPs).

(A) Two-way clustered heatmap of log-transformed primer variant utilization during amplification of fecal genomic DNA. Samples (columns) are color-coded by amplification method (TAS or DePCR) and amplification annealing temperature $\left(40^{\circ}, 45^{\circ}, 50^{\circ}, 55^{\circ}\right.$ and $60^{\circ} \mathrm{C}$ ), with five technical replicates per condition and rarefaction to 1,800 sequences/sample. Primers (rows) are clustered by profile similarity across all samples and represent all 18 primer variants (RPV1 - RPV18) present in the 806R degenerate primer pool. Theoretical melting temperatures for each primer are shown adjacent to primer name. (B) mMDS ordination of PUPs based on Bray-Curtis similarity. Vectors represent Pearson correlations (>0.9) for each primer variant. Ellipses represent $95 \%$ confidence intervals around centroids for DePCR amplification reactions. Five technical replicates per condition were generated and for each sample, rarefaction was performed to 1,800 sequences. (C) Regression analysis was performed was performed on average Shannon index values for primer utilization for each methodology (TAS and DePCR) across annealing temperature. A very small effect of annealing temperature on primer utilization evenness was observed in TAS (orange line). A negative quadratic relationship was observed between annealing temperature and primer utilization evenness in DePCR (blue line). Analyses were based on five technical replicates rarefied to 4,500 sequences per sample. 
A $\quad$ Peerd

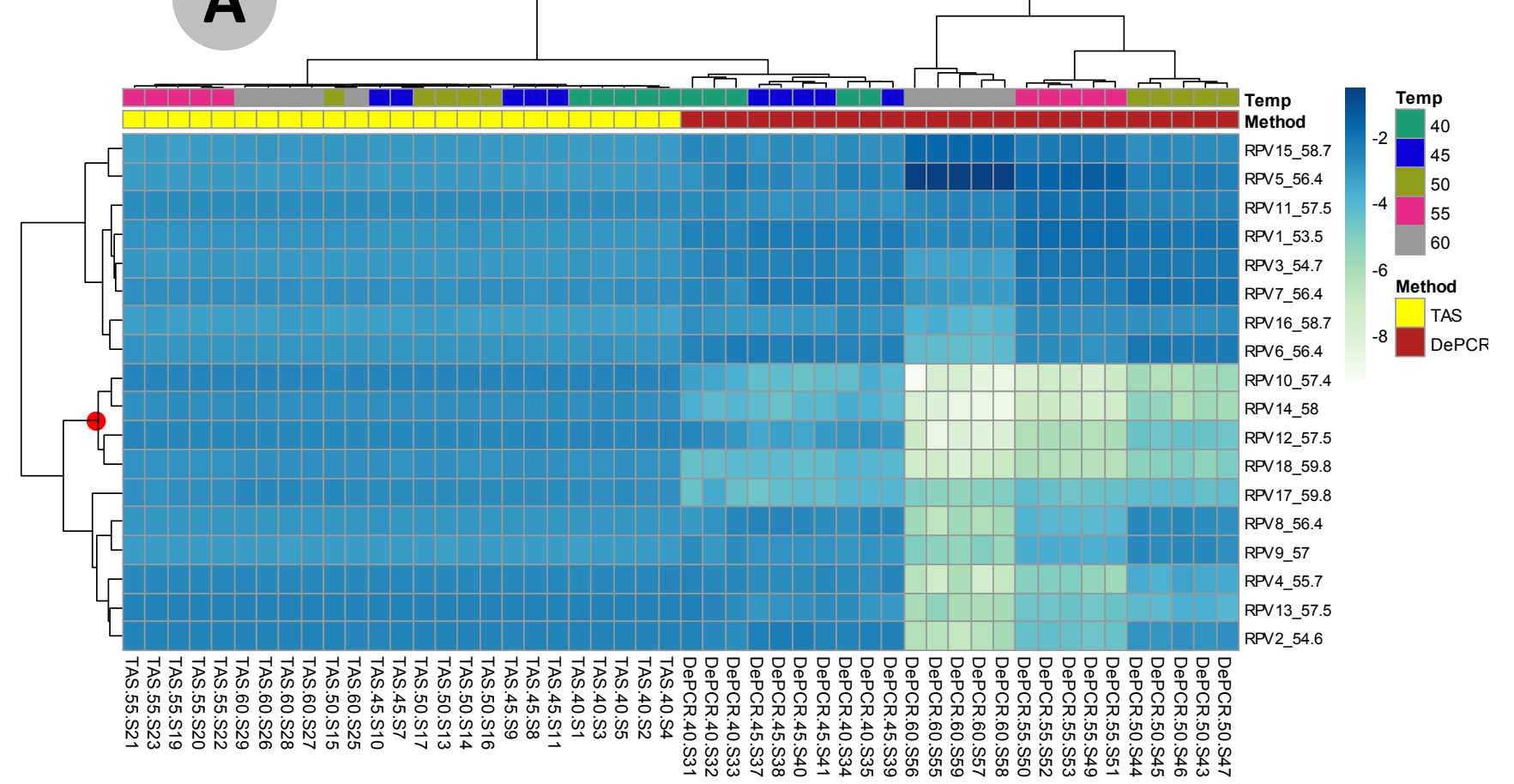

C

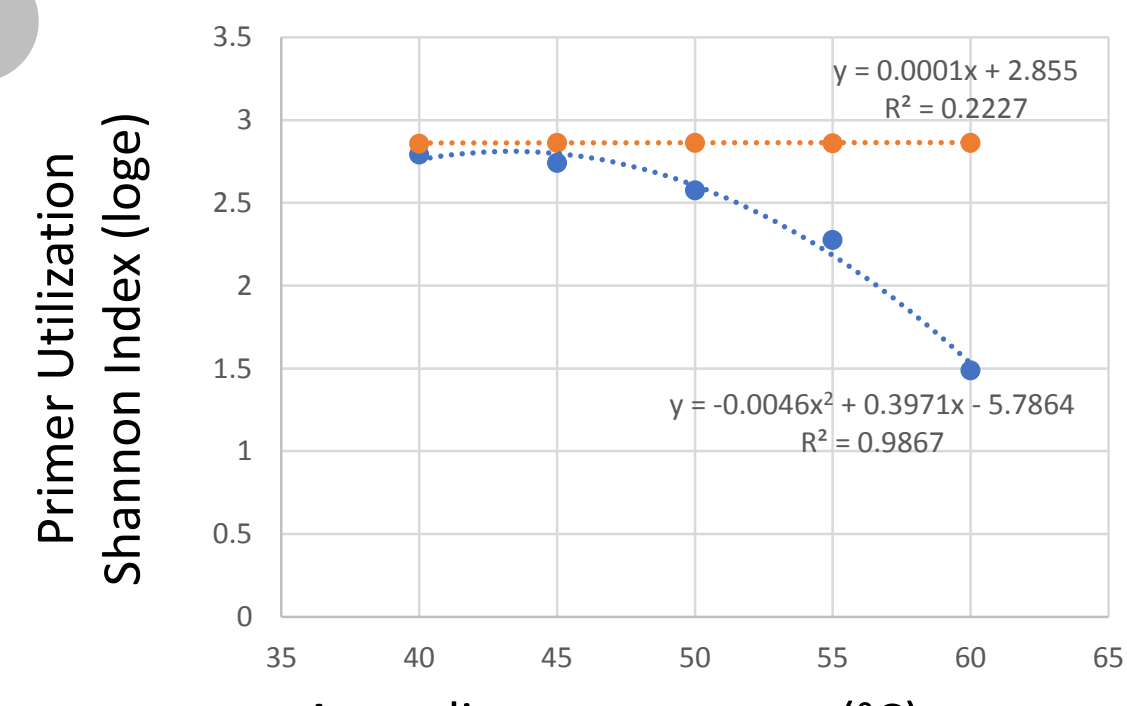

Annealing temperature $\left({ }^{\circ} \mathrm{C}\right.$ )eer] reviewing PDF | (2019:02:34879:20:NNEW 30 Apr 2019)

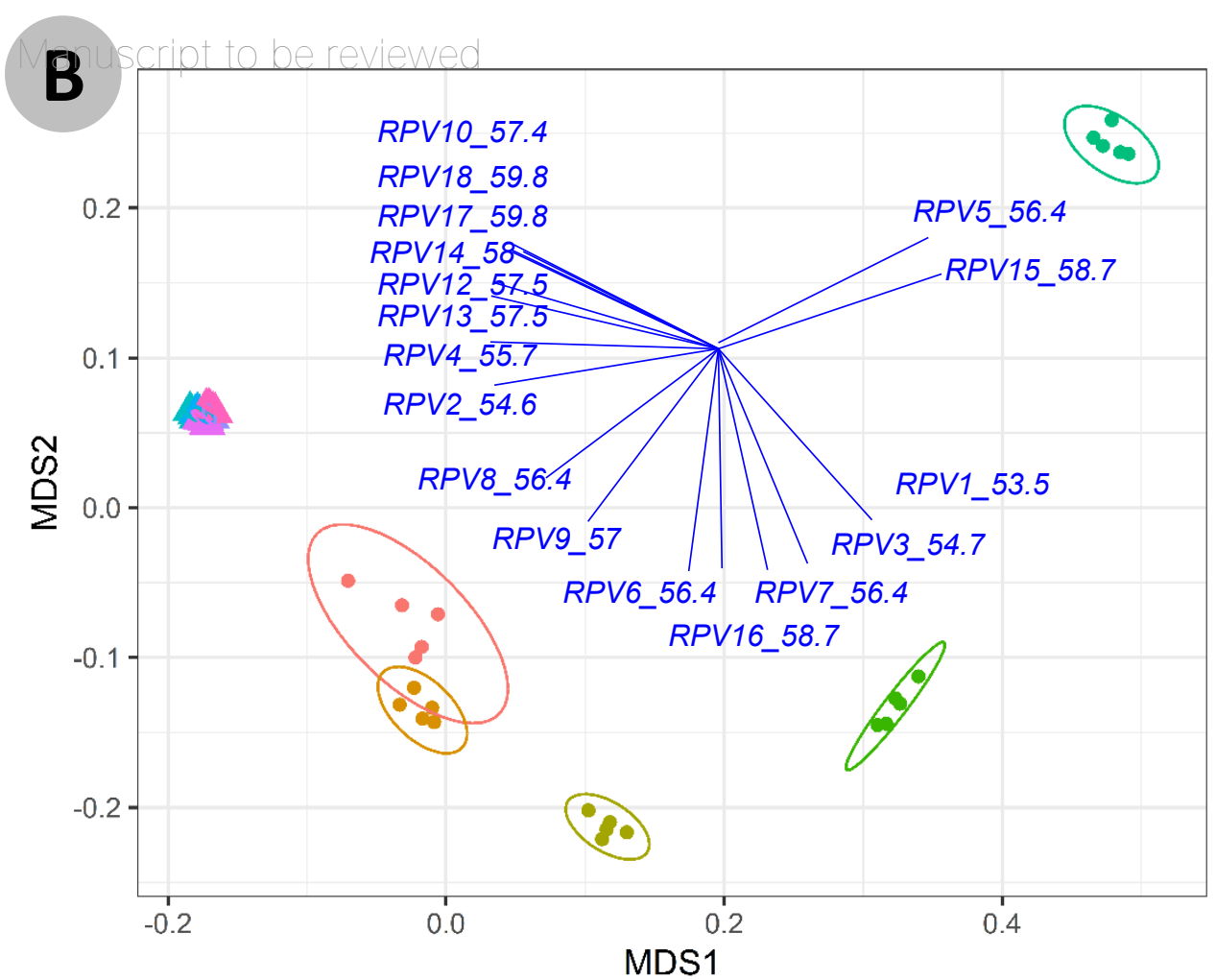

Combined

$\rightarrow$ DePCR40

-- DePCR45

$\rightarrow$ DePCR50

$\rightarrow$ DePCR55

$\rightarrow$ DePCR60

$\rightarrow$ TAS40

$\rightarrow$ TAS45

$\rightarrow$ TAS50

$\rightarrow$ TAS55

$\rightarrow$ TAS60

Method

- DePCR

$\Delta$ TAS 


\section{Figure 5}

Microbial community structure revealed using individual primer variants with TAS and DePCR amplification methodologies.

(A) Fecal gDNA was amplified using the 341F primer with18 unique $806 \mathrm{R}$ reverse primer variants (RPVs) under standard PCR (TAS) and DePCR workflows. Three RPVs were removed from the TAS analysis due to pipetting error, as described in the text. Genus-level biological observation matrices (BIOMs) were visualized using mMDS. Each amplification with a unique RPV was performed in technical duplicate, and five technical replicates were generated using degenerate primer pools (TAS_pool or DePCR_pool). All samples were rarefied to 1,800 sequences. Ellipses represent 95\% confidence intervals around centroids. TAS profiles generated with RPVs were significantly distinct from TAS profiles generated with degenerate primer pools ( $A N O S I M R=0.487 ; P=0.003$ ). DePCR profiles generated with RPVs were not significantly distinct from DePCR profiles generated with degenerate primer pools (ANOSIM $R=-0.306 ; P=0.99$ ). (B) Within-group Bray-Curtis dissimilarity distributions for profiles generated with RPVs and with degenerate pools. (C) Between-group Bray-Curtis dissimilarity distributions for observed microbial community structure generated with RPVs and with degenerate primer pools. Average dissimilarity among TAS_pool and TAS RPV profiles (0.211) was greater than for DePCR_pool and DePCR RPV profiles (0.154) (ANOVA P<0.001). 

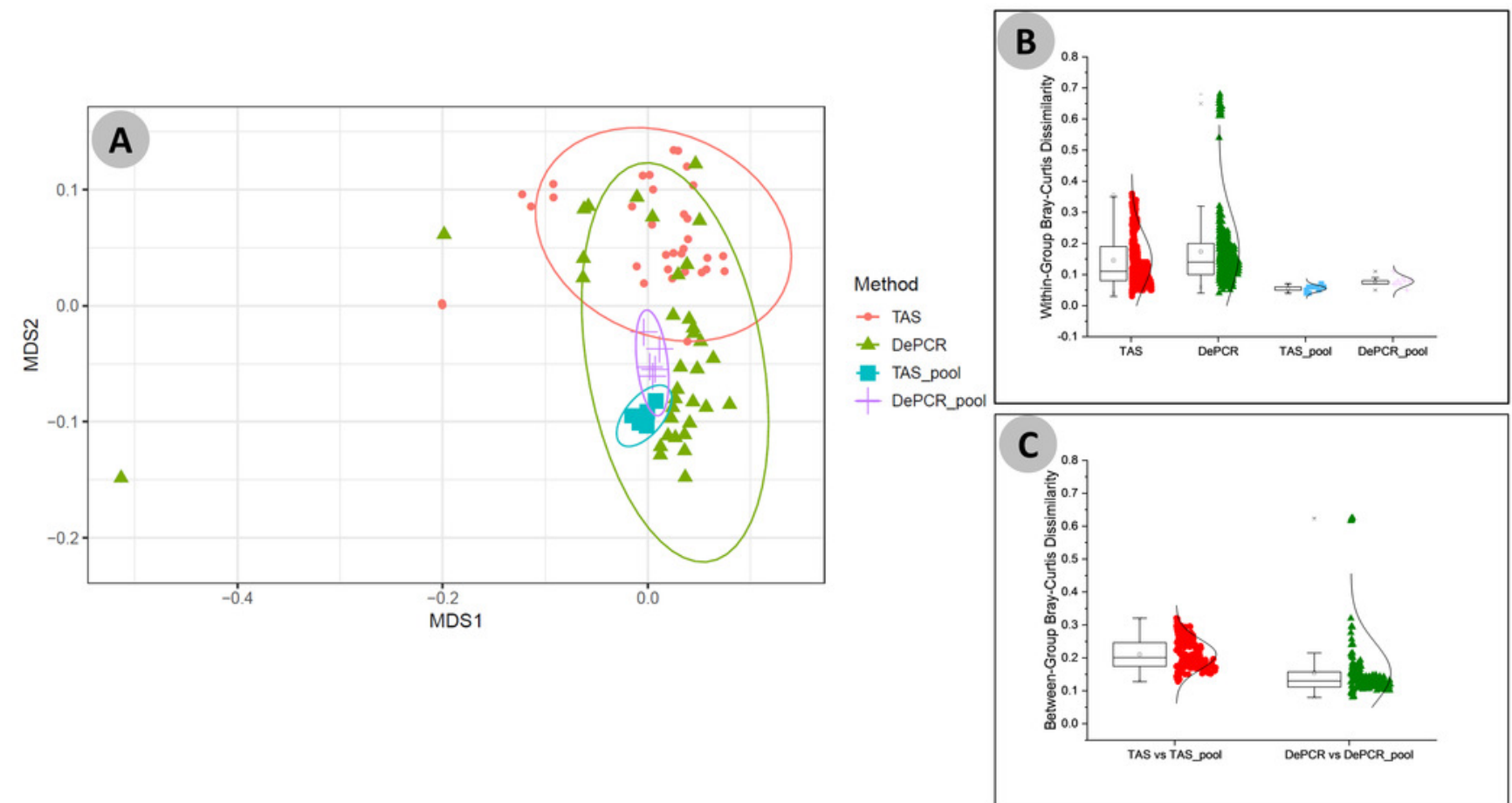


\section{Table $\mathbf{1}$ (on next page)}

Primers used in this study.

Locus-specific primers were synthesized with linker sequences to allow for two-stage PCR amplification and incorporation of sample-specific barcodes, as described in the text. Primer $806 \mathrm{R}$ is 18 -fold degenerate, and variants were synthesized as a pool as well as individually. Access Array primer sequences, synthesized by Fluidigm (PE1-CS1 and PE2-[BC]-CS2), are shown in Figure 2. 


\begin{tabular}{|c|c|c|c|c|}
\hline 341F Primer & Primer Sequence & Linker (CS1) Sequence & Final Sequence Name & Final Sequence Ordered \\
\hline $341 \mathrm{~F}$ & CCTACGGGAGGCAGCAG & ACACTGACGACATGGTTCTACA & >CS1_515F & ACACTGACGACATGGTTCTACACCTACGGGAGGCAGCAG \\
\hline
\end{tabular}

\begin{tabular}{|c|c|c|c|c|}
\hline $\begin{array}{l}\text { 806R Primer and } \\
\text { Variants }\end{array}$ & Primer Sequence & Linker (CS2) Sequence & Final Sequence Name & Final Sequence Ordered \\
\hline $806 \mathrm{R}$ & GGACTACHVGGGTWTCTAAT & TACGGTAGCAGAGACTTGGTCT & $>C S 2 \_806 R$ & TACGGTAGCAGAGACTTGGTCTGGACTACHVGGGTWTCTAAT \\
\hline 806R-RPV1 & GGACTACTAGGGTATCTAAT & TACGGTAGCAGAGACTTGGTCT & >CS2_806R_V1 & TACGGTAGCAGAGACTTGGTCTGGACTACTAGGGTATCTAAT \\
\hline 806R-RPV2 & GGACTACTAGGGTTTCTAAT & TACGGTAGCAGAGACTTGGTCT & $>C S 2 \_806 R \_V 2$ & TACGGTAGCAGAGACTTGGTCTGGACTACTAGGGTTTCTAAT \\
\hline 806R-RPV3 & GGACTACAAGGGTATCTAAT & TACGGTAGCAGAGACTTGGTCT & $>C S 2 \_806 R \_V 3$ & TACGGTAGCAGAGACTTGGTCTGGACTACAAGGGTATCTAAT \\
\hline 806R-RPV4 & GGACTACAAGGGTTTCTAAT & TACGGTAGCAGAGACTTGGTCT & $>C S 2 \_806 R \_V 4$ & TACGGTAGCAGAGACTTGGTCTGGACTACAAGGGTTTCTAAT \\
\hline 806R-RPV5 & GGACTACCAGGGTATCTAAT & TACGGTAGCAGAGACTTGGTCT & $>C S 2 \_806 R \_V 5$ & TACGGTAGCAGAGACTTGGTCTGGACTACCAGGGTATCTAAT \\
\hline 806R-RPV6 & GGACTACAGGGGTATCTAAT & TACGGTAGCAGAGACTTGGTCT & $>C S 2 \_806 R \_V 6$ & TACGGTAGCAGAGACTTGGTCTGGACTACAGGGGTATCTAAT \\
\hline 806R-RPV7 & GGACTACTGGGGTATCTAAT & TACGGTAGCAGAGACTTGGTCT & $>C S 2 \_806 R \_V 7$ & TACGGTAGCAGAGACTTGGTCTGGACTACTGGGGTATCTAAT \\
\hline 806R-RPV8 & GGACTACTCGGGTATCTAAT & TACGGTAGCAGAGACTTGGTCT & $>C S 2 \_806 R \_V 8$ & TACGGTAGCAGAGACTTGGTCTGGACTACTCGGGTATCTAAT \\
\hline 806R-RPV9 & GGACTACACGGGTATCTAAT & TACGGTAGCAGAGACTTGGTCT & $>C S 2 \_806 R \_V 9$ & TACGGTAGCAGAGACTTGGTCTGGACTACACGGGTATCTAAT \\
\hline 806R-RPV10 & GGACTACTCGGGTTTCTAAT & TACGGTAGCAGAGACTTGGTCT & $>C S 2 \_806 R \_V 10$ & TACGGTAGCAGAGACTTGGTCTGGACTACTCGGGTTTCTAAT \\
\hline 806R-RPV11 & GGACTACCAGGGTTTCTAAT & TACGGTAGCAGAGACTTGGTCT & >CS2_806R_V11 & TACGGTAGCAGAGACTTGGTCTGGACTACCAGGGTTTCTAAT \\
\hline 806R-RPV12 & GGACTACAGGGGTTTCTAAT & TACGGTAGCAGAGACTTGGTCT & $>C S 2$ 806R_V12 & TACGGTAGCAGAGACTTGGTCTGGACTACAGGGGTTTCTAAT \\
\hline 806R-RPV13 & GGACTACTGGGGTTTCTAAT & TACGGTAGCAGAGACTTGGTCT & $>C S 2 \_806 R \_V 13$ & TACGGTAGCAGAGACTTGGTCTGGACTACTGGGGTTTCTAAT \\
\hline 806R-RPV14 & GGACTACACGGGTTTCTAAT & TACGGTAGCAGAGACTTGGTCT & >CS2_806R_V14 & TACGGTAGCAGAGACTTGGTCTGGACTACACGGGTTTCTAAT \\
\hline 806R-RPV15 & GGACTACCGGGGTATCTAAT & TACGGTAGCAGAGACTTGGTCT & $>C S 2 \_806 R \_V 15$ & TACGGTAGCAGAGACTTGGTCTGGACTACCGGGGTATCTAAT \\
\hline 806R-RPV16 & GGACTACCCGGGTATCTAAT & TACGGTAGCAGAGACTTGGTCT & $>C S 2 \_806 R \_V 16$ & TACGGTAGCAGAGACTTGGTCTGGACTACCCGGGTATCTAAT \\
\hline 806R-RPV17 & GGACTACCGGGGTTTCTAAT & TACGGTAGCAGAGACTTGGTCT & $>C S 2 \_806 R \_V 17$ & TACGGTAGCAGAGACTTGGTCTGGACTACCGGGGTTTCTAAT \\
\hline 806R-RPV18 & GGACTACCCGGGTTTCTAAT & TACGGTAGCAGAGACTTGGTCT & >CS2_806R_V18 & TACGGTAGCAGAGACTTGGTCTGGACTACCCGGGTTTCTAAT \\
\hline
\end{tabular}

\begin{tabular}{|c|l|l|l|l|}
\hline Illumina Primers & & & & Final Sequence Ordered \\
\hline P5 & & & & AATGATACGGCGACCACCGA \\
\hline P7 & & & & CAAGCAGAAGACGGCATACGA \\
\hline
\end{tabular}




\section{Table 2 (on next page)}

Rates of detectable chimeras in sequence data.

Average rates of detectable chimeras are shown for each experiment performed in this study. Significantly lower rates of chimera formation were observed for DePCR-amplified gDNA samples relative to TAS-amplified samples, across multiple annealing temperatures. No significant difference in chimera formation was observed with DePCR methodology with varying gDNA input levels. Significantly higher chimera formation was also observed with TAS relative to DePCR when individual primer variants (RPVs) were utilized. SD = standard deviation. 


\begin{tabular}{|c|c|c|c|c|c|}
\hline Experiment & $\begin{array}{c}\text { PCR } \\
\text { Method }\end{array}$ & $\begin{array}{l}\text { Annealing } \\
\text { Temp. }\left({ }^{\circ} \mathrm{C}\right)\end{array}$ & $\begin{array}{c}\text { Input } \\
\text { concentration } \\
\text { (ng/reaction) }\end{array}$ & $\begin{array}{l}\text { Chimera } \\
\text { detection rate } \\
\text { [Average }(S D)]\end{array}$ & ANOVA \\
\hline \multirow{10}{*}{$\begin{array}{l}\text { Annealing } \\
\text { temperature }\end{array}$} & TAS & 40 & 10 & $5.16 \%(0.37 \%)$ & \multirow{2}{*}{$1.41 \mathrm{E}-09$} \\
\hline & DePCR & 40 & 10 & $0.05 \%(0.03 \%)$ & \\
\hline & TAS & 45 & 10 & $6.49 \%(0.29 \%)$ & \multirow{2}{*}{$4.05 \mathrm{E}-11$} \\
\hline & DePCR & 45 & 10 & $0.10 \%(0.07 \%)$ & \\
\hline & TAS & 50 & 10 & $6.53 \%(0.21 \%)$ & \multirow{2}{*}{$2.02 \mathrm{E}-12$} \\
\hline & DePCR & 50 & 10 & $0.04 \%(0.02 \%)$ & \\
\hline & TAS & 55 & 10 & $5.69 \%(0.39 \%)$ & \multirow{2}{*}{$9.66 \mathrm{E}-10$} \\
\hline & DePCR & 55 & 10 & $0.05 \%(0.02 \%)$ & \\
\hline & TAS & 60 & 10 & $5.46 \%(0.49 \%)$ & \multirow{2}{*}{ 7.56E-09 } \\
\hline & DePCR & 60 & 10 & $0.03 \%(0.02 \%)$ & \\
\hline \multirow{5}{*}{$\begin{array}{l}\text { Input gDNA } \\
\text { concentration }\end{array}$} & DePCR & 50 & 20 & $0.05 \%(0.02 \%)$ & \multirow{5}{*}{$5.20 \mathrm{E}-01$} \\
\hline & DePCR & 50 & 10 & $0.03 \%(0.03 \%)$ & \\
\hline & DePCR & 50 & 5 & $0.03 \%(0.01 \%)$ & \\
\hline & DePCR & 50 & 2.5 & $0.02 \%(0.01 \%)$ & \\
\hline & DePCR & 50 & 1.25 & $0.03 \%(0.03 \%)$ & \\
\hline \multirow{2}{*}{$\begin{array}{l}\text { Reverse primer } \\
\text { variants }\end{array}$} & TAS & 50 & 10 & $11.98 \%$ (3.85\%) & \multirow{2}{*}{0.00} \\
\hline & DePCR & 50 & 10 & $0.06 \%(0.08 \%)$ & \\
\hline
\end{tabular}

1 


\section{Table 3 (on next page)}

Alpha diversity indices of observed microbial communities.

Shannon indices were calculated at the taxonomic levels of genus for all samples amplified using TAS and DePCR methodologies across five annealing temperatures of $40^{\circ}, 45^{\circ}, 50^{\circ}$, $55^{\circ}$ and $60^{\circ} \mathrm{C}$. Datasets were rarefied to 4,500 sequences/sample. For each methodology and annealing temperature, an average and standard deviation of five technical replicates is shown. At all temperatures, TAS-amplified samples had higher Shannon indices relative to DePCR-amplified samples. SD = standard deviation. 


\begin{tabular}{|c|c|c|c|c|c|}
\hline $\begin{array}{c}\text { PCR } \\
\text { Method }\end{array}$ & $\begin{array}{l}\text { Annealing } \\
\text { Temp. }\left({ }^{\circ} \mathrm{C}\right)\end{array}$ & $\begin{array}{l}\text { Shannon Index } \\
\text { [Average (SD)] }\end{array}$ & ANOVA & $\begin{array}{c}\text { Richness } \\
\text { [Average (SD)] }\end{array}$ & ANOVA \\
\hline TAS & 40 & $2.69(0.02)$ & \multirow{2}{*}{$4.76 \mathrm{E}-05$} & $61.20(1.92)$ & \multirow{2}{*}{$1.92 \mathrm{E}-05$} \\
\hline DePCR & 40 & $2.55(0.03)$ & & $50.60(1.82)$ & \\
\hline TAS & 45 & $2.72(0.03)$ & \multirow{2}{*}{$5.86 \mathrm{E}-05$} & $60.60(2.70)$ & \multirow{2}{*}{$1.32 \mathrm{E}-01$} \\
\hline DePCR & 45 & $2.59(0.03)$ & & $57.20(3.63)$ & \\
\hline TAS & 50 & $2.74(0.03)$ & \multirow{2}{*}{$2.58 \mathrm{E}-04$} & $64.00(2.65)$ & \multirow{2}{*}{$6.56 \mathrm{E}-02$} \\
\hline DePCR & 50 & $2.66(0.01)$ & & $59.60(3.78)$ & \\
\hline TAS & 55 & $2.72(0.02)$ & \multirow{2}{*}{$8.13 E-04$} & $62.00(1.87)$ & \multirow{2}{*}{$2.98 \mathrm{E}-02$} \\
\hline DePCR & 55 & $2.64(0.03)$ & & $58.60(2.19)$ & \\
\hline TAS & 60 & $2.72(0.01)$ & \multirow{2}{*}{$6.16 \mathrm{E}-04$} & $60.60(2.70)$ & \multirow{2}{*}{$3.31 \mathrm{E}-02$} \\
\hline DePCR & 60 & $2.63(0.03)$ & & $56.60(2.19)$ & \\
\hline
\end{tabular}

1 


\section{Table 4 (on next page)}

Effects of amplification method and reverse primer variants on observed microbial community alpha diversity.

Fecal gDNA was PCR amplified with 18-fold degenerate reverse primer pools (5 technical replicates), and with each unique reverse primer variant (RPV; 2 technical replicates). Data sets were rarefied to 1,800 sequences per sample, and Shannon indices (loge) were calculated. When using fully degenerate primer pools, average Shannon index was significantly higher for TAS methodology relative to DePCR methodology. When data from all reactions with individual RPVs were analyzed, average Shannon index was significantly lower for TAS methodology relative to DePCR methodology. Data from RPVs (1,800 sequences/sample) were pooled and re-rarefied to 1,800 sequences ( 5 repetitions), and the resulting average Shannon index was significantly lower for the TAS methodology relative to DePCR methodology. Different approaches with the DePCR method did not generate significantly different Shannon indices (ANOVA $P=0.377$ ), while the same approaches generated significantly different Shannon indices (ANOVA $P<0.001$ ). 


\begin{tabular}{|c|c|c|c|c|}
\hline Comparison & \# replicates analyzed & $\begin{array}{c}\text { Average Shannon } \\
\text { Index (SD), TAS }\end{array}$ & $\begin{array}{c}\text { Average Shannon } \\
\text { Index (SD), DePCR }\end{array}$ & ANOVA \\
\hline $\begin{array}{c}\text { Amplification with 18-fold degenerate } \\
\text { primer pools }\end{array}$ & 5 & $2.71(0.03)$ & $2.66(0.04)$ & $3.14 \mathrm{E}-05$ \\
\hline Amplification with each RPV independently & 33 (TAS) or 36 (DePCR) & $2.4(0.01)$ & $2.58(0.21)$ & $5.95 \mathrm{E}-05$ \\
\hline $\begin{array}{c}\text { Summation of independent RPVs and re- } \\
\text { rarefaction to 1800 sequences (5x) }\end{array}$ & 5 & $2.48(0.03)$ & $2.69(0.02)$ & $7.40 \mathrm{E}-07$ \\
\hline
\end{tabular}

